\title{
Sirtuin 7 promotes non-small cell lung cancer progression by facilitating G1/S phase and epithelial-mesenchymal transition and activating AKT and ERK1/2 signaling
}

\author{
YINGYING ZHAO $^{1 *}$, XIA YE ${ }^{1 *}$, RUIFANG CHEN $^{1}$, QIAN GAO $^{1}$, DAGUO ZHAO ${ }^{2}$,

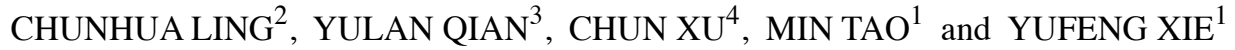 \\ Departments of ${ }^{1}$ Oncology, ${ }^{2}$ Respiratory Medicine, ${ }^{3}$ Pharmacy and ${ }^{4}$ Cardio-Thoracic Surgery, \\ The First Affiliated Hospital of Soochow University, Suzhou, Jiangsu 215006, P.R. China
}

Received November 26, 2019; Accepted May 29, 2020

DOI: $10.3892 /$ or.2020.7672

\begin{abstract}
Increasing evidence has indicated the roles of sirtuin 7 (SIRT7) in numerous human cancers. However, the effects and the clinical significance of SIRT7 in human lung cancer is largely unknown. The present research demonstrated that SIRT7 was increased in human lung cancer tumor tissues. SIRT7 upregulation was associated with clinicopathological characteristics of lung cancer malignancy including positive lymph node metastasis, high pathologic stage and large tumor size. SIRT7 was also upregulated in human non-small cell lung cancer (NSCLC) cell lines. Furthermore SIRT7-overexpressed A549 (A549-SIRT7) and SIRT7-knocked down H292 (H292-shSIRT7) human NSCLC cell lines were established. Using these NSCLC cells and xenograft mouse models, it was revealed that SIRT7 overexpression markedly promoted growth and $\mathrm{G} 1$ to $\mathrm{S}$ cell cycle phase transition as well as migration, invasion and distant lung metastasis in A549 NSCLC cells, whereas SIRT7 knockdown suppressed these processes in H292 NSCLC cells. Mechanistically, in A549 NSCLC cells, SIRT7 overexpression significantly activated not only protein kinase B (AKT) signaling but also extracellular signal-regulated kinase 1/2 (ERK1/2) signaling. SIRT7 overexpression also significantly downregulated cyclin-dependent kinase (CDK) inhibitors including p21 and p27 as well as upregulated cyclins including cyclin D1 and cyclin E1, and CDKs including CDK2 and CDK4. Notably, the epithelial-mesenchymal transition
\end{abstract}

Correspondence to: Professor Yufeng Xie or Dr Min Tao, Department of Oncology, The First Affiliated Hospital of Soochow University, 188 Shizi Street, Suzhou, Jiangsu 215006, P.R. China

E-mail: sdxyf@163.com

E-mail: taomin@suda.edu.cn

${ }^{*}$ Contributed equally

Key words: non-small cell lung cancer, sirtuin 7, cell cycle, epithelial-mesenchymal transition, protein kinase B, extracellular signal-regulated kinase $1 / 2$
(EMT) process of A549 NSCLC cells was facilitated by SIRT7 overexpression, as evidenced by E-cadherin epithelial marker downregulation and mesenchymal markers (N-cadherin, vimentin, Snail and Slug) upregulation. In addition, SIRT7 knockdown in H292 NSCLC cells exhibited the opposite regulatory effects. Moreover, inhibition of AKT signaling abated the promoting effects of SIRT7 in NSCLC cell proliferation and EMT progression. The present data indicated that SIRT7 accelerated human NSCLC cell growth and metastasis possibly by promotion of G1 to S-phase transition and EMT through modulation of the expression of G1-phase checkpoint molecules and EMT markers as well as activation of AKT and ERK1/2 signaling. SIRT7 could be an innovative potential target for human NSCLC therapy.

\section{Introduction}

The number of deaths from lung cancer has been increasing exponentially over the recent years worldwide (1). This malignant tumor is mainly caused by environmental pollution and smoking (2). Up to $85 \%$ of all lung cancer cases are of the NSCLC subtype, which is furthermore classified into adenocarcinoma, squamous-cell carcinoma and large-cell carcinoma (3). Therapeutic techniques for NSCLC include surgery, targeted therapy, chemotherapy, radiotherapy, immunotherapy, or their combination (2). In spite of recent developments in diagnostic and therapeutic approaches of NSCLC, the prognosis of NSCLC patients is still unsatisfactory $(3,4)$. Therefore, identifying the potential molecular targets and defining the underlying molecular mechanisms involved in NSCLC progression and metastasis are key to discovering the effective targets to improve its prognosis.

Sirtuins consisting of sirtuin 1-7 (SIRT1-7) members belong to the $\mathrm{NAD}^{+}$-dependent histone/protein deacetylase family $(5,6)$. Sirtuins bear a highly conserved $\mathrm{NAD}^{+}$-binding and catalytic core domain but display diverse deacetylation and ADP-ribosylation enzymatic activities, substrate proteins and cellular localizations and functions (5-7). Sirtuins have been previously reported to participate in a number of cellular processes including senescence, metabolism, proliferation, differentiation, apoptosis, DNA repair, genomic 
stability, chromatin remodeling, gene transcription and post-translational modification $(5,6,8)$. Of all the sirtuins, SIRT7 is a newly discovered member. SIRT7 can promote ribosome biogenesis via inducing synthesis of ribosomal RNA and transfer RNA (9-11). Notably, SIRT7 is a highly selective acetylated histone $\mathrm{H} 3$ lysine 18 (H3K18Ac) deacetylase and SIRT7-mediated deacetylation of H3K18Ac maintains oncogenic transformation by transcriptionally suppressing expression of tumor suppressor genes (12). Accumulating evidence (13-22) has demonstrated that SIRT7 is upregulated and functions as an oncogene in most cancers including breast, thyroid, colorectal and gastric cancer, hepatocellular carcinoma, cholangiocarcinoma, prostate cancer, bladder cancer and angiosarcoma. Furthermore, high expression of SIRT7 has been revealed to contribute to tumor cell resistance to chemotherapy and radiotherapy $(20,23,24)$. Conversely, SIRT7 has been demonstrated to be downregulated in the oral, neck and head squamous cell carcinoma and pancreatic cancer (25-27). SIRT7 can attenuate tumor cell EMT and metastasis through deacetylating mothers against decapentaplegic homolog 4 (SMAD4) to antagonize transforming growth factor- $\beta$ (TGF- $\beta$ ) signaling $(27,28)$. These studies indicate that SIRT7 exerts tumor-promoting or tumor-suppressive properties.

Several studies have revealed that SIRT7 plays key roles in human lung cancer $(29,30)$. SIRT7 was revealed to be upregulated in human NSCLC cells and its depletion could inhibit NSCLC cell growth by activating the pro-apoptotic signaling pathway (29). miR-3666 suppressed NSCLC cell growth by targeting SIRT7 (29). SIRT7 depletion could further sensitize NSCLC cells to gemcitabine chemotherapy via inhibiting autophagy (30). However, the effects and the clinical roles of SIRT7 in lung cancer are less understood. The present study detected the expression of SIRT7 in clinical tissues of human lung cancer and NSCLC cells, and analyzed the clinical association between SIRT7 expression of lung cancer tissues and its clinicopathological characteristics. The roles of SIRT7 overexpression or knockdown in growth and metastasis of NSCLC cells were determined and the related molecular mechanism was elucidated.

\section{Materials and methods}

Cellular and molecular biological reagents. Dulbecco's modified Eagle's medium (DMEM) was supplied by HyClone; GE Healthcare Life Sciences. Fetal bovine serum (FBS) and puromycin were supplied by Gibco; Thermo Fisher Scientific, Inc. Penicillin-streptomycin antibiotics, the NheI and $S g s \mathrm{I}$ restriction enzymes and the reverse transcription kit were supplied by Thermo Fisher Scientific, Inc. Taq DNA polymerase, dNTPmix, T4 DNA ligase and the RNA extraction kit were supplied by TaKaRa Biotechnology Co., Ltd. SYBR Green Master was supplied from Roche Applied Science. Lipofectamine 2000 was supplied by Invitrogen; Thermo Fisher Scientific, Inc. Blasticidin S was supplied by Yeasen Biotechnology Co., Ltd. Cell Counting Kit-8 (CCK-8) was supplied by Beijing Solarbio Science \& Technology Co., Ltd. Propidium iodide (PI)/RNase Staining Buffer Solution was supplied by BD Biosciences. The bicinchoninic acid protein assay kit, the mammalian cell lysis buffer for western blotting and the SuperEnhanced chemiluminescence detection
(BeyoECL Star) kit were supplied by Beyotime Institute of Biotechnology. The diaminobenzidine substrate was supplied by Boster Biological Technology. The primers were supplied by Sangon Biotech Co., Ltd.

Antibodies. Primary antibody rabbit anti-SIRT7 (cat. no. 12994-1-AP) was supplied by ProteinTech Group, Inc. Rabbit anti-AKT (cat. no. AF6261), anti-ERK1/2 (cat. no. AF0155), anti-p-AKT (Thr308) (T308) (cat.no. AF3262), anti-p-AKT (Ser473) (S473) (cat.no. AF0016) and anti-p-ERK1/2 (Thr202/Tyr204) (cat. no. AF1015) primary antibodies were supplied by Affinity Biosciences. Primaryantibodiesincluding rabbitanti- $\beta$-actin(cat.no.YM3214), anti-p21 (cat. no. YT3497), anti-p27 (cat. no. YT3502), anti-cyclinD1 (cat.no. YT1173), anti-cyclinE1 (cat.no. YT1176), anti-CDK2 (cat. no. YT0832) and anti-CDK4 (cat. no. YT5198) were supplied by ImmunoWay Biotechnology Company. Primary antibodies including rabbit anti-E-cadherin (product no. 3195), anti-N-cadherin (product no. 13116), anti-Vimentin (product no. 5741), anti-Snail (Snail1) (product no. 3879) and anti-Slug (Snail2) (product no. 9585) for western blotting were supplied by Cell Signaling Technology, Inc. Primary antibody rabbit anti-Snail (cat. no. 101167-T10) for immunohistochemistry was supplied by Sino Biological, Inc. Primary antibody rabbit anti-Slug (cat. no. GTX128796) for immunohistochemistry was supplied by GeneTex, Inc. Secondary antibody horseradish peroxidase (HRP)-conjugated anti-rabbit IgG (product no. 7074 for western blotting and product no. BM3894 for immunohistochemistry) was supplied by Cell Signaling Technology, Inc. and Boster Biological Technology, respectively.

Plasmid and lentiviral vectors. The pGEM/SIRT7 cloning plasmid carrying human SIRT7 coding sequence (CDS) (BC017305) was supplied by Sino Biological, Inc. Plasmids including pLenti6.3/IRES/GFP lentiviral plasmid-expressing blasticidin S deaminase and green fluorescent protein (GFP) as well as pLP1, pLP2 and VSVG lentiviral packing plasmids were supplied by Novobio Scientific, Inc. Lentiviruses including control shRNA lentivirus (LV-shcontrol) and SIRT7 shRNA (h) lentivirus (LV-shSIRT7) containing puromycin resistance gene were supplied by Santa Cruz Biotechnology, Inc.

Cell culture. The human embryonic kidney cell line 293T, the normal human bronchial epithelial cell line HBEpiC and the human NSCLC cell lines including H292, A549, H1299 and H1975 were supplied by the Cell Bank of Type Culture Collection of the Chinese Academy of Sciences. Cells were grown in culture medium (DMEM containing 10\% FBS and $100 \mathrm{U} / \mathrm{ml}$ of penicillin-streptomycin antibiotics), identified by short tandem repeat (STR) profiling (Genetic Testing Biotechnology) and confirmed to have no mycoplasma contamination. 293T cells were cultured for 2 weeks before transfected with plasmids for the construction of lentiviral vectors, whereas HBEpiC, H292, A549, H1299 and H1975 cells were cultured for 1 week for the following RT-qPCR, western blotting and infection experiments. The culture medium supplemented with $10 \mu \mathrm{g} / \mathrm{ml}$ of blasticidin $\mathrm{S}$ was used to grow SIRT7-overexpressed A549-SIRT7 and control A549-mock NSCLC cell lines. The culture medium supplemented with 
$2 \mu \mathrm{g} / \mathrm{ml}$ of puromycin was used to grow SIRT7-silenced H292-shSIRT7 and control H292-shcontrol NSCLC cell lines. After antibiotic selection for about 4 weeks, stable transgenic cell lines were established and frozen at once. For in vitro assays, the frozen transgenic cells were thawed and cultured for 2 weeks to be amplified. The cells were then used for RT-qPCR and western blotting assays as well as in vitro functional assays including CCK-8, colony formation, cell cycle, wound healing and Transwell migration/invasion. The culture duration of each in vitro functional assay was subsequently specified. For in vivo animal experiments, the transgenic cells were thawed and cultured for 3 weeks to be amplified before being injected into nude mice. The aforementioned cells were cultured in a humidified chamber at $37^{\circ} \mathrm{C}$ under $5 \% \mathrm{CO}_{2}$ to obtain enough cells for each of the following experiments.

Nude mice. Forty-eight four-week-old female athymic BALB/c nude mice (average weight, $15 \mathrm{~g}$ ) were supplied by Shanghai Laboratory Animal Center. The mice were maintained in the animal facility at Soochow University (Suzhou, China) under specific pathogen-free conditions with a 12-h light/dark cycle at $22 \pm 2^{\circ} \mathrm{C}$ and $60 \pm 5 \%$ humidity, provided with free access to food and water. All animal experiments were approved by the Animal Research Ethics Committee of Soochow University (IRB no. A201809059).

Lung cancer tissue specimens. We obtained 102 pairs of human lung cancer tumor tissues and adjacent non-tumor lung tissues (collected at a distance of more than $6 \mathrm{~cm}$ from the tumor site) derived from 102 lung cancer patients (age range, 39-83 years; 52 male and 50 female patients) at the Department of Cardio-Thoracic Surgery of the First Affiliated Hospital of Soochow University (Suzhou, China) from January 2016 to April 2017. The patients had undergone lung cancer surgery but had not received any neoadjuvant chemotherapy or radiotherapy. The collected tissue samples were fixed in $10 \%$ neutral formalin for $24 \mathrm{~h}$ at room temperature, and subsequently embedded in paraffin. Two experienced pathologists independently performed the pathological staging of tumors. The medical history of patients was reviewed to describe their clinicopathological features. The present study was conducted after approval by the Ethics Committee of the First Affiliated Hospital of Soochow University (IRB no. 2016128). Signed informed consent was obtained from all the participants.

Tissue microarray (TMA) and section preparation. After tissue localization by hematoxylin and eosin (H\&E) analysis, the aforementioned paired lung cancer tumor tissue and adjacent non-tumor lung tissue specimens fixed with formalin and embedded in paraffin were used for preparation of human lung cancer TMA with a sample diameter of $1.5 \mathrm{~mm}$ (102 cases, 102 pairs, 204 dots). The TMA was then cut into $3 \mu \mathrm{m}$-thick sections for subsequent immunohistochemistry analysis.

Construction and titration of lentiviral vectors. Amplification of CDS fragment (1203 bp) of human SIRT7 was performed by polymerase chain reaction (PCR) with pGEM/SIRT7 plasmid template and human full-length SIRT7 CDS-specific primer pair (SIRT7-F1: 5'-CTAGCTAGCGCCACCATG GCAGCCGGGGGTCTGAG-3' and SIRT7-R1: 5'-TTGGCG
CGCCTTACGTCACTTTCTTCCTTTTTGTGCG-3'). It was then inserted into the lentiviral plasmid pLenti6.3/IRES/GFP between NheI and SgsI sites to generate a recombinant lentiviral plasmid pLenti6.3/SIRT7/IRES/GFP. The 293T cells were seeded in $10-\mathrm{cm}$ dishes at a density of $2 \times 10^{6}$ cells $/ 10 \mathrm{ml}$ medium/dish and cultured overnight. Subsequently, the plasmid pLenti6.3/SIRT7/IRES/GFP or pLenti6.3/IRES/GFP was co-transfected into 293 T cells with pLP1, pLP2 and VSVG by Lipofectamine 2000 . The cells were cultured for another $48 \mathrm{~h}$ at $37^{\circ} \mathrm{C}$. The lentivirus expressing SIRT7 (LV-SIRT7) and the blank lentivirus (LV) were consequently produced and concentrated by ultracentrifugation. The lentiviral biological titre $(\mathrm{TU} / \mathrm{ml})$ was determined based on the sum of GFP-positive $293 \mathrm{~T}$ cells as observed by fluorescence microscopy.

Establishment of stable cell lines. The A549 NSCLC cell line was infected with LV-SIRT7 or LV (control) at a multiplicity of infection (MOI) of 50 and selected with blasticidin $\mathrm{S}(10 \mu \mathrm{g} / \mathrm{ml})$, leading to generation of A549-SIRT7 (SIRT7-overexpressed) or A549-mock (control) transgenic cell line. The H292 NSCLC cell line was infected with LV-shSIRT7 or LV-shcontrol (control) (50 MOI) and selected with puromycin $(2 \mu \mathrm{g} / \mathrm{ml})$, resulting in formation of H292-shSIRT7 (SIRT7-silenced) or H292-shcontrol (control) transgenic cell line. The transgene efficiency in A549 NSCLC cells was analyzed using the GFP reporter by fluorescence microscopy and flow cytometry. The efficiency of SIRT7 overexpression or knockdown in A549 or H292 NSCLC cells, respectively, was analyzed by reverse transcription-quantitative PCR (RT-qPCR) and western blotting.

$R T$ - $q P C R$. The total RNAs of A549, H292, H1299, H1975, HBEpiC, A549-SIRT7, A549-mock, H292-shSIRT7 and H292-shcontrol cells were purified by a MiniBEST universal RNA extraction kit following the supplier's instructions and reversely-transcribed to first-strand cDNAs with a RevertAid RT Reverse Transcription kit. Subsequently, qPCR analysis of human SIRT7 mRNA expression was performed by SYBR-Green Master using human SIRT7-specific primer pair (SIRT7-F2: 5'-ACTTGGTCGTCTACACAGGC-3' and SIRT7-R2: 5'-GGTGATGCTCATGTGGGTGA-3', product size: $158 \mathrm{bp}$ ) ( $\beta$-actin used as an internal control) as previously described (31). RT was conducted at $42^{\circ} \mathrm{C}$ for $60 \mathrm{~min}$, followed by $70^{\circ} \mathrm{C}$ for $5 \mathrm{~min}$. qPCR amplification was conducted at $95^{\circ} \mathrm{C}$ for $300 \mathrm{sec}$ followed by 40 cycles at $95^{\circ} \mathrm{C}$ for $10 \mathrm{sec}$ and $60^{\circ} \mathrm{C}$ for $30 \mathrm{sec}$. SIRT7 mRNA expression level in A549, H292, H1299 and H1975 NSCLC cells (HBEpiC used as a cell control); SIRT7-overexpressed A549-SIRT7 NSCLC cells (A549-mock used as a cell control); and SIRT7-silenced H292-shSIRT7 NSCLC cells (H292-shcontrol used as a cell control) was standardized to $\beta$-actin expression and computed via the $2^{-\Delta \Delta \mathrm{Cq}}$ method (32), respectively.

CCK-8 assay. In 96-well plates, the A549-SIRT7 vs. A549-mock and H292-shSIRT7 vs. H292-shcontrol human NSCLC cells ( $1 \times 10^{4}$ cells $/ 200 \mu 1$ medium) were seeded per well. At the 1st, 2nd, 3rd and 4th day following cell culture, the tumor cell vitality was evaluated by CCK-8 (10 $\mu \mathrm{l} /$ well). Using an automatic microplate reader at $450 \mathrm{~nm}$, the optical density (OD) was read. The growth curve of tumor cells in vitro was plotted as the changes in OD value over culture time. 
Colony formation assay. In 6-well plates, the A549-SIRT7 vs. A549-mock and H292-shSIRT7 vs. H292-shcontrol human NSCLC cells (200 cells/2 ml medium) were seeded per well. After being incubated for two weeks, the colonies of tumor cells were fixed with $4 \%$ paraformaldehyde for $15 \mathrm{~min}$ followed by staining in $0.5 \%$ crystal violet for $20 \mathrm{~min}$ at room temperature. Colonies of $>10$ cells were counted under a white light field using fluorescence microscope IX51 (Olympus Corporation; magnification, x200). Analysis of the relative clonogenic ability of these tumor cells was then carried out by GraphPad Prism 6 software (GraphPad, Inc.).

Cell cycle assay. After being cultured in 6-well plates, the A549-SIRT7 vs. A549-mock and H292-shSIRT7 vs. H292-shcontrol human NSCLC cells $\left(0.5 \times 10^{6}\right.$ cells $)$ were collected and incubated overnight at $4^{\circ} \mathrm{C}$ in ice-cold $70 \%$ ethanol. The PI/RNase Staining Buffer Solution (500 $\mu \mathrm{l}$ per reaction) was then used to stain the cells in the dark for 30 min. Finally, the cell cycle was detected using CytoFLEX flow cytometer (Beckman Coulter, Inc.) and analyzed with CXP analysis software 2.2 (Beckman Coulter, Inc.).

Wound healing assay. In 6-well plates, the A549-SIRT7 vs. A549-mock and H292-shSIRT7 vs. H292-shcontrol human NSCLC cells $\left(5 \times 10^{5}\right.$ cells $/ 5 \mathrm{ml}$ medium) were seeded per well. Upon almost $100 \%$ confluence of cells, scratches were generated and reference points were marked on the outer bottom of 6 -well plates nearby. Cellular debris was removed from the wells by rinsing with fresh medium. To maintain cell growth, the wells were supplemented with DMEM containing 2\% FBS. The migration of tumor cells was studied by microscopic observation (white light field, fluorescence microscope IX51; magnification, x100) at 0,24 and $48 \mathrm{~h}$ after wounding. The capability of migration of tumor cells was quantitatively analyzed by ImageJ $1.52 \mathrm{v}$ software (National Institutes of Health).

Transwell migration/invasion assay. In $8 \mu \mathrm{m}$-pore size 24-well Transwell filters (EMD Millipore), the A549-SIRT7 vs. A549-mock and H292-shSIRT7 vs. H292-shcontrol human NSCLC cells with a density of $2 \times 10^{4}$ or $2 \times 10^{5}$ cells $/ 100 \mu \mathrm{l}$ serum-free medium were placed in the upper chamber without or with $50 \mu \mathrm{l}$ Matrigel (1:8 pre-diluted with serum-free medium) (Corning, Inc.) for Transwell migration or invasion assays, respectively. The lower chamber was supplemented with culture medium. After $24 \mathrm{~h}$, the tumor cells which had migrated or invaded into bottom side were placed in $4 \%$ paraformaldehyde to be fixed for $15 \mathrm{~min}$ and $0.5 \%$ crystal violet for $20 \mathrm{~min}$ at room temperature to be stained. Microscopy (white light field, fluorescence microscope IX51; magnification, $\mathrm{x} 200$ ) was then used to assess the migratory and invasive abilities of tumor cells.

Tumor xenograft mouse models. For establishment of a tumor subcutaneous xenograft model, the A549-SIRT7 vs. A549-mock and H292-shSIRT7 vs. H292-shcontrol human NSCLC cells ( $2 \times 10^{6}$ cells per mouse; 6 mice per group) were injected subcutaneously into the right flanks of nude mice. The growth of tumors was tracked by monitoring the tumor volume every week until 4 weeks after inoculation of tumor cells and the weight 4 weeks after inoculation of tumor cells. Tumor diameter was measured by a Vernier calliper every week and the tumor volume was calculated using a formula: Volume $\left(\mathrm{mm}^{3}\right)=\mathrm{a}^{2} \mathrm{~b} / 2$ ( $\mathrm{a}$ is the shortest diameter, whereas $\mathrm{b}$ is the longest diameter). Four weeks after subcutaneous injection, the tumor-bearing mice were humanely euthanized by continuous inhalation with $30 \% \mathrm{CO}_{2}$ for $5 \mathrm{~min}$. Death was confirmed when mice had no heartbeat for $30 \mathrm{sec}$ accompanied by no response to the toe pinch reflex. Subsequently, the xenograft tumors were removed and weighed by an electronic analytical balance. The xenograft tumor-derived sections ( $3 \mu \mathrm{m}$-thick) were then prepared and used for subsequent immunohistochemical analysis. For establishment of a tumor lung metastasis model, the aforementioned tumor cells ( $2 \times 10^{6}$ cells per mouse; 6 mice per group) were injected intravenously into the tail veins of nude mice. Six weeks after intravenous injection, the mice were euthanized as aforementioned to harvest their lung tissues. The lung tissue sections ( $3 \mu \mathrm{m}$-thick) were then prepared and used for $\mathrm{H} \& \mathrm{E}$ analysis of lung metastatic nodules.

Western blotting. The protein expression of SIRT7 in A549, H292, H1299, H1975 and HBEpiC cells as well as SIRT7, p-AKT (T308/S473)/total AKT, p-ERK1/2/total ERK1/2, p21/27, cyclin D1/E1, CDK2/4, E/N-cadherin, vimentin, Snail and Slug in A549-SIRT7, A549-mock, H292-shSIRT7 and H292-shcontrol cells was analyzed by western blotting ( $\beta$-actin was used as a loading control) as previously reported (31). The primary antibodies and HRP-conjugated anti-rabbit IgG secondary antibody were diluted at 1:1,000 and 1:3,000, respectively. Membranes were incubated with the primary antibodies overnight at $4^{\circ} \mathrm{C}$ overnight and a secondary antibody for $1 \mathrm{~h}$ at room temperature. Protein signals were determined by BeyoECL Star kit, scanned by Gel Imaging System and quantified by ImageJ $1.52 \mathrm{v}$ software (National Institutes of Health).

Immunohistochemistry $(I H C)$. The prepared $3 \mu$ m-thick human lung cancer TMA section was subjected to IHC analysis of SIRT7 using rabbit anti-SIRT7 (1:50) primary antibody and HRP-conjugated anti-rabbit IgG $(1: 1,000)$ secondary antibody as previously described (31). SIRT7 expression level of each specimen was evaluated by a weighted IHC score $(0-1,-$; $2-3,+; 4-5,++$; and 6-7, +++) (31). A weighted score of $\geq 4(++$ or +++$)$ was considered as SIRT7 high expression in a tissue specimen. In addition, the sections derived from A549-SIRT7, A549-mock, H292-shSIRT7 and H292-shcontrol subcutaneous xenograft tumors were subjected to IHC analysis of p-AKT (T308) (1:50), p-AKT (S473) (1:50), AKT (1:50), p-ERK1/2 (1:50), ERK1/2 (1:100), p21 (1:200), p27 (1:100), cyclin D1 (1:100), cyclin E1 (1:100), CDK2 (1:100), CDK4 (1:100), E-cadherin (1:200), N-cadherin (1:125), vimentin (1:100), Snail (1:500) and Slug (1:100), respectively. Briefly, the xenograft tumor-derived sections ( $3 \mu \mathrm{m}$-thick) were fixed in $10 \%$ neutral formalin for $24 \mathrm{~h}$ at room temperature and embedded in paraffin. After deparaffinization, rehydration, rinse, antigen retrieval, quenching of endogenous peroxidase activity with $3 \% \mathrm{H}_{2} \mathrm{O}_{2}$ and blocking with $5 \%$ bovine serum albumin (BSA), the sections were incubated with primary antibodies at $4^{\circ} \mathrm{C}$ overnight and a secondary antibody for $1 \mathrm{~h}$ 


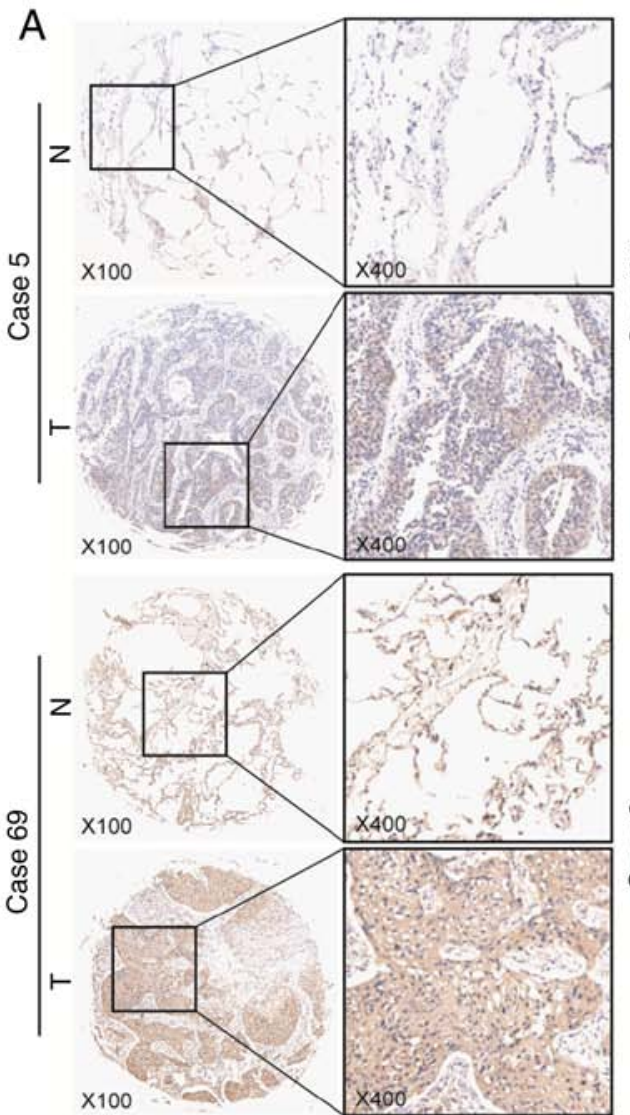

B
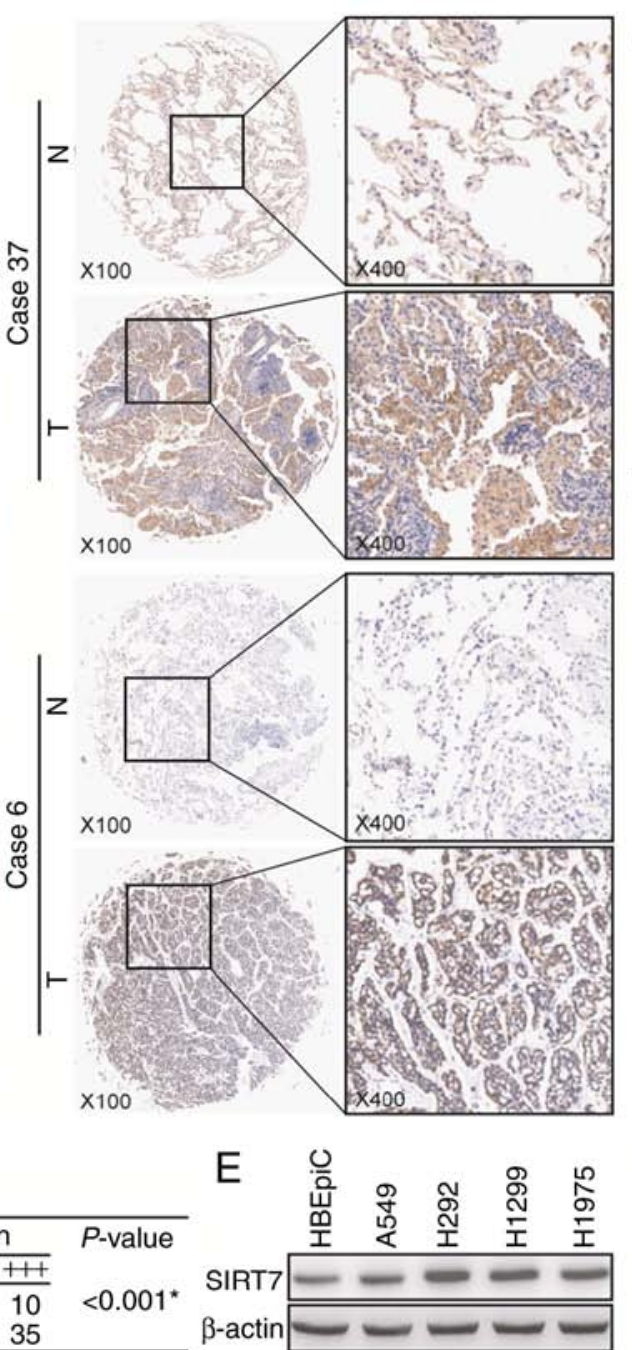

C
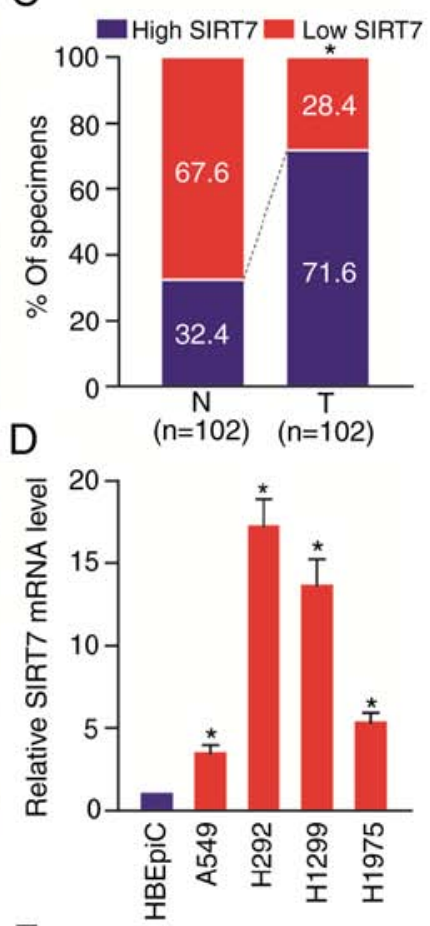

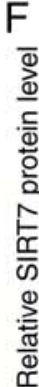

Figure 1. SIRT7 is increased in human lung cancer tissues and cell lines. (A-C) IHC analysis of SIRT7 in human lung cancer TMA. (A) The representative IHC images of Case 5 (tumor tissue IHC score: -), Case 37 (tumor tissue IHC score: +), Case 69 (tumor tissue IHC score: ++ ) and Case 6 (tumor tissue IHC score: +++). (B) The number of IHC scoring -, +, ++ or +++ cases in 102 paired lung cancer tumor/adjacent non-tumor tissues. P<0.001, Mann-Whitney $\mathrm{U}$ test. (C) The percentage of high/low SIRT7 expression in lung cancer tumor/adjacent non-tumor tissues. ${ }^{*} \mathrm{P}<0.05$, Pearson's $\chi^{2}$ test. T, tumor tissue; N, adjacent non-tumor tissue. (D) The relative level of SIRT7 mRNA in human NSCLC cells (HBEpiC served as a control) analyzed by RT-qPCR. (E and F) Analysis of SIRT7 protein in NSCLC cells by western blotting. (E) The representative western blot images. (F) The relative level of SIRT7 protein in NSCLC cells (HBEpiC served as a control). ${ }^{*} \mathrm{P}<0.05$, one-way repeated measures ANOVA with LSD post hoc multiple comparisons, $\mathrm{n}=6$ per group. SIRT7, sirtuin 7 ; IHC, immunohistochemistry; TMS, tissue microarray; NSCLC, non-small cell lung cancer; ANOVA, analysis of variance; LSD, least significant difference.

at room temperature. Finally, the sections were stained with diaminobenzidine tetrahydrochloride (DAB) for $5 \mathrm{~min}$ and then counterstained with $\mathrm{H} \& \mathrm{E}$ for $5 \mathrm{~min}$ at room temperature. Following dehydration and mounting, observation was performed and images were captured under a white light field using fluorescence microscope IX51 (magnification, x100). Protein expression was analyzed by ImageJ $1.52 \mathrm{v}$ software (National Institutes of Health).

AKT inhibition assay. The A549-SIRT7 human NSCLC cells were pre-treated with an allosteric AKT inhibitor MK-2206 (APExBIO) $(10 \mu \mathrm{M})$ or dimethylsulfoxide (DMSO) without MK-2206 (vehicle control) for $1 \mathrm{~h}$. Then the MK-2206- and DMSO-treated A549-SIRT7 cells as well as the untreated A549-SIRT7 and A549-mock cells were subjected to CCK-8, wound healing and Transwell invasion assays. Additionally, the aforementioned cells were cultured for another $24 \mathrm{~h}$ and then subjected to western blot analysis of EMT markers.

Statistical analyses. The data of the IHC scoring of SIRT7 expression in lung cancer tissues were presented as -, +, ++ or +++ and a Mann-Whitney $U$ test was used to assess the difference of the rank data. The categorical data of high or low expression of SIRT7 in lung cancer tissues were presented as the percentage of total cases and a Pearson's $\chi^{2}$ test was used to analyze the differences. The measurement data of the in vitro cell studies and in vivo animal studies were analyzed by a normal distribution test and presented as the mean \pm standard deviation (SD) when $P>0.1$. A Student's t-test was then used for comparisons of differences between two independent groups. 
Table I. The association of SIRT7 expression with lung cancer clinicopathological features.

\begin{tabular}{|c|c|c|c|c|}
\hline Variables & $\begin{array}{l}\text { Total cases } \\
(\mathrm{n}=102)(\%)\end{array}$ & $\begin{array}{l}\text { SIRT7-low expression } \\
\quad(\mathrm{n}=29 ; 28.4 \%)\end{array}$ & $\begin{array}{l}\text { SIRT7-high expression } \\
\quad(\mathrm{n}=73 ; 71.6 \%)\end{array}$ & P-value \\
\hline Age (years) & & & & 0.433 \\
\hline$\leq 65$ & $52(51.0)$ & 13 & 39 & \\
\hline$>65$ & $50(49.0)$ & 16 & 34 & \\
\hline Sex & & & & 0.925 \\
\hline Male & $52(51.0)$ & 15 & 37 & \\
\hline Female & $50(49.0)$ & 14 & 36 & \\
\hline Histology & & & & 0.483 \\
\hline Adenocarcinoma & $76(74.5)$ & 23 & 53 & \\
\hline SCC/Other & $26(25.5)$ & 6 & 20 & \\
\hline Differentiation & & & & 0.069 \\
\hline Well & $8(7.8)$ & 5 & 3 & \\
\hline Moderate/Poor & $94(92.2)$ & 24 & 70 & \\
\hline Tumor size & & & & $<0.001^{\mathrm{a}}$ \\
\hline $\mathrm{T} 1$ & $44(43.1)$ & 21 & 23 & \\
\hline $\mathrm{T} 2 / \mathrm{T} 3 / \mathrm{T} 4$ & $58(56.9)$ & 8 & 50 & \\
\hline Pathologic stage & & & & $0.036^{\mathrm{a}}$ \\
\hline $\mathrm{I} / \mathrm{II}$ & $77(75.5)$ & 26 & 51 & \\
\hline III/IV & $25(24.5)$ & 3 & 22 & \\
\hline Lymph node metastasis & & & & $0.012^{\mathrm{a}}$ \\
\hline No & $69(67.6)$ & 25 & 44 & \\
\hline $\mathrm{N} 1 / \mathrm{N} 2$ & $33(32.4)$ & 4 & 29 & \\
\hline
\end{tabular}

${ }^{\mathrm{a}} \mathrm{P}<0.05$, Pearson's $\chi^{2}$ test. SIRT7, sirtuin 7.

After further testing homogeneity of variance $(\mathrm{P}>0.1$ indicated homogeneity of variance), one-way or two-way repeated measures analysis of variance (ANOVA) with least significant difference (LSD) post hoc multiple comparisons were used to analyze the differences among groups. All aforementioned statistical tests were performed with SPSS17.0 (SPSS, Inc.). A two-sided value of $\mathrm{P}<0.05$ was considered to indicate a statistically significant difference.

\section{Results}

SIRT7 is increased in human lung cancer tissues and cell lines. For determination of the expression level of SIRT7 in human lung cancer clinical tissues, SIRT7 expression in human lung cancer TMA (102 pairs of lung cancer tumor tissue and adjacent non-tumor tissue specimens) was analyzed by IHC (Fig. 1A). Among these lung cancer tumor tissues, high SIRT7 expression was observed in $71.6 \%$ of the cases (73 cases; 35 cases scored ' +++ ' and 38 cases scored ' ++ ') and low SIRT7 expression was detected in $28.4 \%$ of the cases (29 cases; 18 cases scored '+' and 11 cases scored '-') (Fig. 1B and C). Whereas in the matched adjacent non-tumor tissues, there were only $32.4 \%$ of cases (33 cases; 10 cases scored ' +++ ' and 23 cases scored ' ++ ') exhibiting high SIRT7 expression and $67.6 \%$ of cases ( 69 cases; 39 cases scored ' + ' and 30 cases scored '-') showing low SIRT7 expression (Fig. 1B and C).
The data demonstrated that SIRT7 was significantly increased in the human lung cancer tumor tissues by comparison with the adjacent non-tumor control tissues $(\mathrm{P}<0.05)$ (Fig. 1A-C). Subsequently, the level of SIRT7 in human NSCLC cell lines including A549, H292, H1299 and H1975 were detected by RT-qPCR (Fig. 1D) and western blot (Fig. 1E and F) analyses, revealing that these 4 types of human NSCLC cell lines also expressed a significantly higher level of SIRT7 than HBEpiC control cell line $(\mathrm{P}<0.05)$. These results revealed that SIRT7 is elevated in human lung cancer tissues and cell lines.

Upregulation of SIRT7 is correlated with malignant clinicopathological features in human lung cancer. Based on SIRT7 expression level of lung cancer tumor tissues, 29 patients with low SIRT7 expression ('+' and '-') in tumor tissues were classified as the SIRT7-low expression group, and 73 patients with high SIRT7 expression ('+++' and '++') in tumor tissues were classified as the SIRT7-high expression group. The association between high or low SIRT7 expression in lung cancer tissues and clinicopathological variables including age, sex, histology, differentiation, tumor size, pathologic stage and lymph node metastasis was then assessed (Table I). The data revealed that the SIRT7 expression level was positively associated with malignant clinicopathological characteristics. A high level of SIRT7 was associated with large tumor size, high pathologic stage 

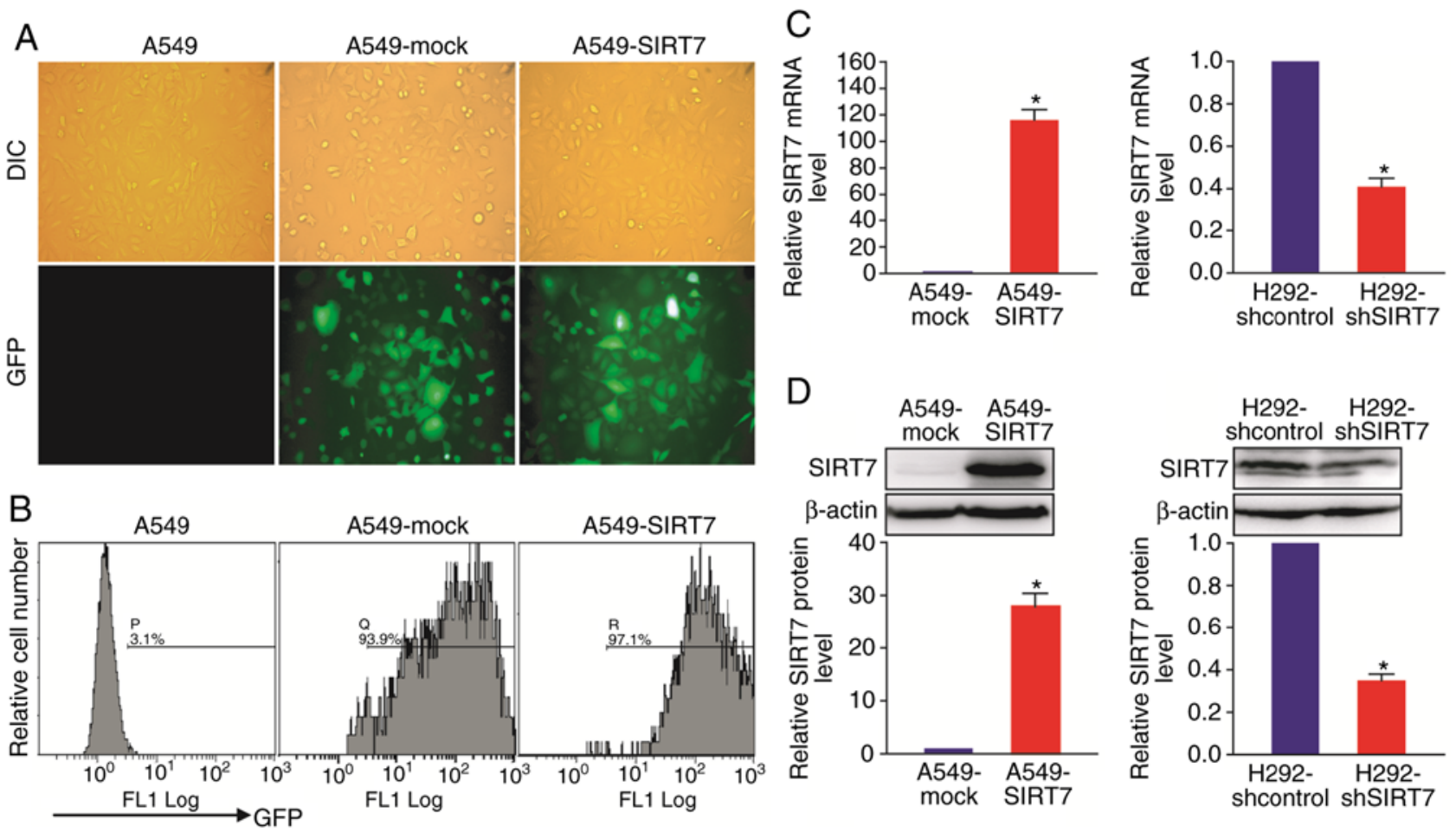

Figure 2. Overexpression/knockdown of SIRT7 in human NSCLC cells. (A) GFP analysis by fluorescence microscopy. (B) GFP analysis by flow cytometry. (C) SIRT7 overexpression or knockdown efficiency detected by RT-qPCR. (D) SIRT7 overexpression or knockdown efficiency detected by western blotting. The representative western blot images are presented in the upper panel. The relative level of SIRT7 protein in A549-SIRT7 (A549-mock served as a control) and H292-shSIRT7 (H292-shcontrol served as a control) NSCLC cells are presented in the lower panel. "P<0.05, Student's $t$-test, $\mathrm{n}=6$ per group. SIRT7, sirtuin 7; NSCLC, non-small cell lung cancer; GFP, green fluorescent protein; reverse transcription-quantitative PCR.

and presence of lymph node metastasis $(\mathrm{P}<0.05)$, indicating that increased expression SIRT7 may be involved in the progression and metastasis of human lung cancer.

Forced expression or knockdown of SIRT7 in human NSCLC cells. To generate a SIRT7-overexpressed lung cancer cell line, LV-SIRT7 or LV (control) lentivirus infected into the A549 NSCLC cell line (relative low expression of SIRT7) and stable cell lines were selected by blasticidin S. According to the fluorescence microscopic (Fig. 2A) and flow cytometric (Fig. 2B) detection, nearly all (more than 90\%) of the LV-SIRT7- or LV-infected A549 (A549-SIRT7 or A549-mock) NSCLC cells expressed GFP, demonstrating a high efficiency of transgene expression. The lentivirus-directed SIRT7 overexpression efficiency in A549 cells was then determined using RT-qPCR and western blot analyses, and it was revealed that both the mRNA (Fig. 2C) and protein (Fig. 2D) levels of SIRT7 in A549-SIRT7 cells were increased compared with A549-mock control cells $(\mathrm{P}<0.05)$. To establish a SIRT7-silenced lung cancer cell line, the H292 NSCLC cell line (relative high expression of SIRT7) was infected with LV-shSIRT7 or LV-shcontrol (control) lentivirus and selected by puromycin. Results of RT-qPCR (Fig. 2C) and western blotting (Fig. 2D) further demonstrated that compared with LV-shcontrol-infected H292 (H292-shcontrol) control cells, LV-shSIRT7-infected H292 (H292-shSIRT7) cells exhibited a reduction in mRNA and protein expression of SIRT7 $(\mathrm{P}<0.05)$. These data revealed that SIRT7-overexpressed A549 and SIRT7-silenced H292 human NSCLC cell lines were successfully obtained.
SIRT7 promotes human NSCLC cell growth and Gl to S phase transition. To address whether SIRT7 influences human NSCLC cell growth, a CCK-8 assay was used to assess the proliferation and viability of A549-SIRT7 vs. A549-mock and H292-shSIRT7 vs. H292-shcontrol human NSCLC cells in vitro. As revealed in Fig. 3A, overexpression of SIRT7 significantly promoted A549 NSCLC cell growth in vitro, whereas knockdown of SIRT7 suppressed H292 NSCLC cell growth in vitro $(\mathrm{P}<0.05)$. Moreover, colony formation assay (Fig. 3B and C) demonstrated that A549-SIRT7 NSCLC cells formed larger and more colonies than A549-mock control cells, whereas H292-shSIRT7 NSCLC cells generated smaller and less colonies than $\mathrm{H} 292$-shcontrol control cells $(\mathrm{P}<0.05)$. The results indicated that SIRT7 can enhance clonogenicity of human NSCLC cells. To further determine whether SIRT7 could accelerate growth of human NSCLC cells in vivo, a human NSCLC subcutaneous xenograft model was established by injecting A549-SIRT7 vs. A549-mock or H292-shSIRT7 vs. H292-shcontrol cells into athymic BALB/c nude mice. The tumor growth including tumor volume and weight of the aforementioned cells was monitored. The in vivo data (Fig. 3D-F) also demonstrated that overexpression of SIRT7 significantly promoted A549 subcutaneous xenograft tumor growth, whereas knockdown of SIRT7 significantly inhibited H292 subcutaneous xenograft tumor growth $(\mathrm{P}<0.05)$. To examine the cellular mechanism by which SIRT7 promotes NSCLC cell growth, a cell cycle assay was performed and A549-SIRT7 vs. A549-mock and H292-shSIRT7 vs. H292-shcontrol NSCLC cells were analyzed by flow cytometry. According to Fig. 3G and H, in A549 cells, overexpression of SIRT7 
A

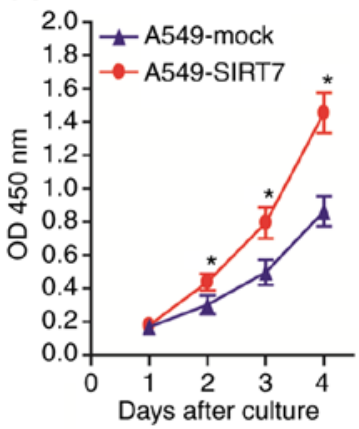

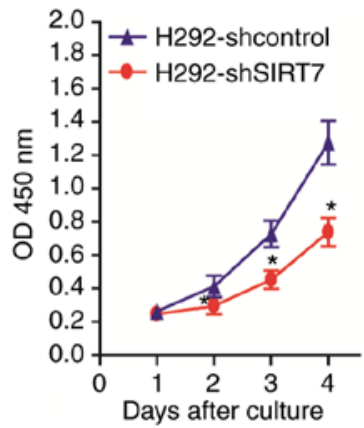

B

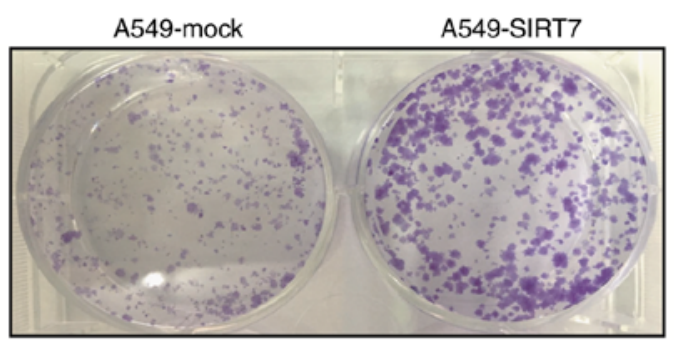

D
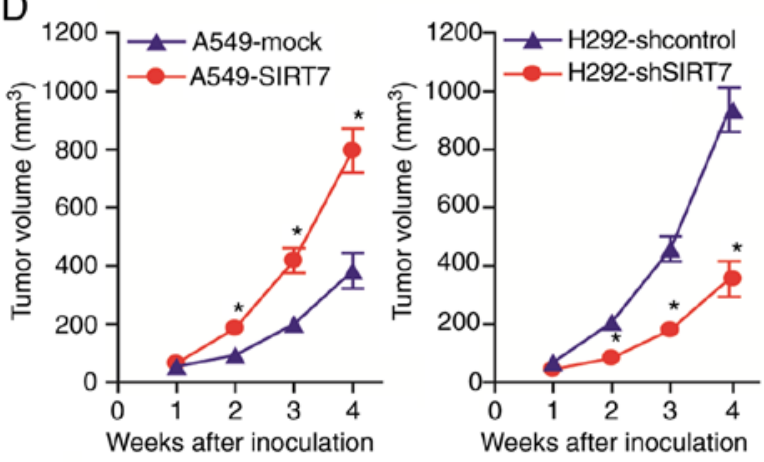

$\mathrm{F}$
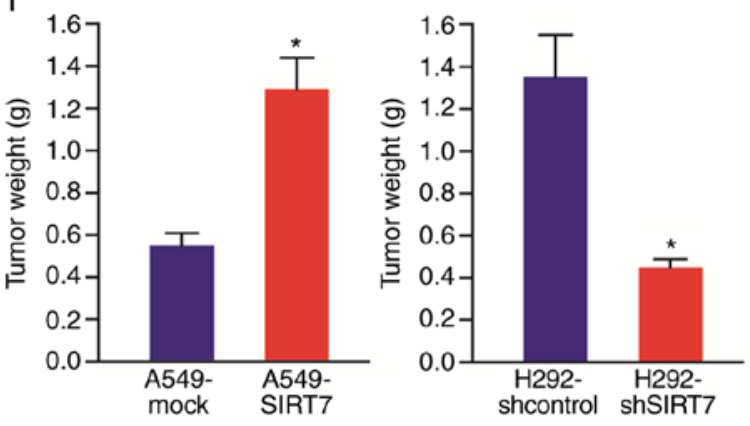

C

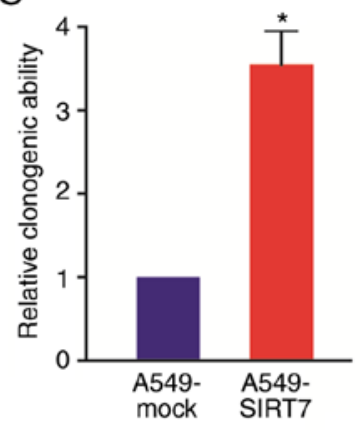

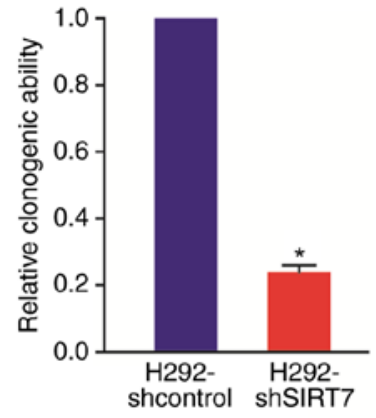

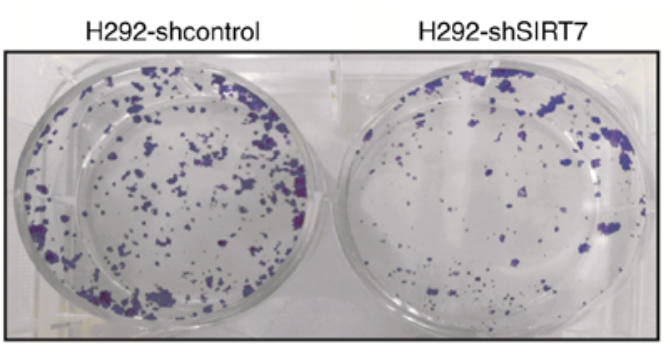

E

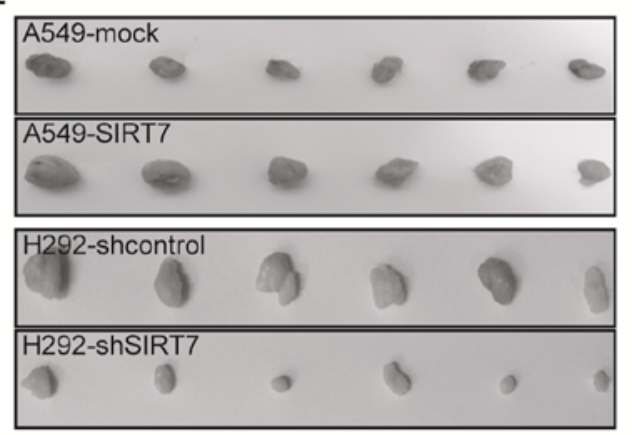

$\mathrm{H}$

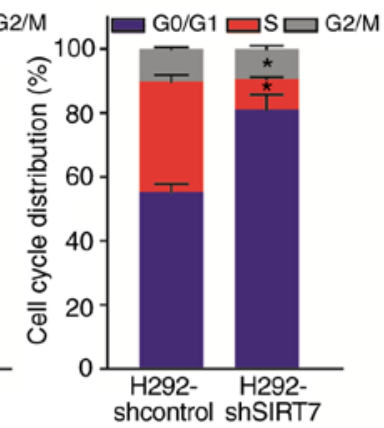

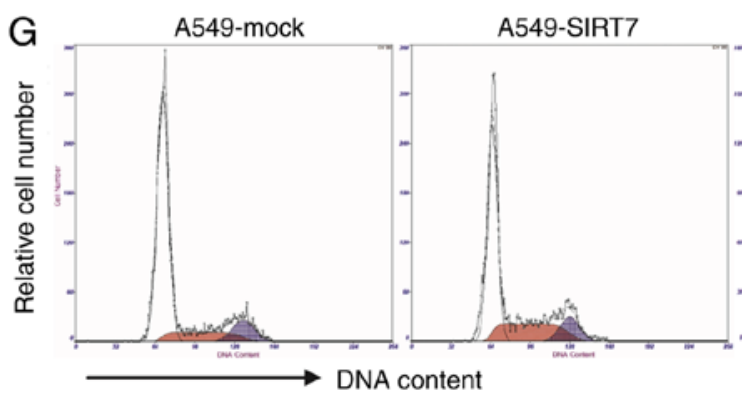

H292-shcontrol

H292-shSIRT7

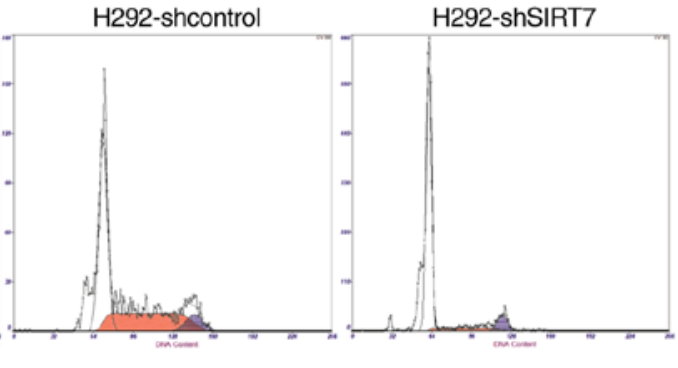

Figure 3. SIRT7 promotes growth and G1 to S-phase transition of human NSCLC cells. (A) CCK-8 analysis of NSCLC cell proliferation/growth. "P<0.05, two-way repeated measures ANOVA with LSD post hoc multiple comparisons, $\mathrm{n}=6$ per group. (B and C) Colony formation assay. (B) The representative images. (C) The relative clonogenic ability of A549-SIRT7 (A549-mock served as a control) and H292-shSIRT7 (H292-shcontrol served as a control) NSCLC cells. " $\mathrm{P}<0.05$, Student $t$-test, $\mathrm{n}=6$ per group. (D-F) Tumor subcutaneous xenograft mouse model. (D) Tumor volume. " $\mathrm{P}<0.05$, two-way repeated measures ANOVA with LSD post hoc multiple comparisons, $\mathrm{n}=6$ per group. (E) The xenograft tumor images. (F) Tumor weight. " $\mathrm{P}<0.05$, Student's $t$-test, $\mathrm{n}=6$ per group. $(\mathrm{G}$ and $\mathrm{H})$ Cell cycle analysis by flow cytometry. (G) The representative images. $(\mathrm{H})$ The percentage of each cell cycle phase. "P<0.05, Student's $t$-test, $\mathrm{n}=6$ per group. SIRT7, sirtuin 7; NSCLC, non-small cell lung cancer; CCK-8, Cell Counting Kit-8; ANOVA, analysis of variance; LSD, least significant difference. 
A

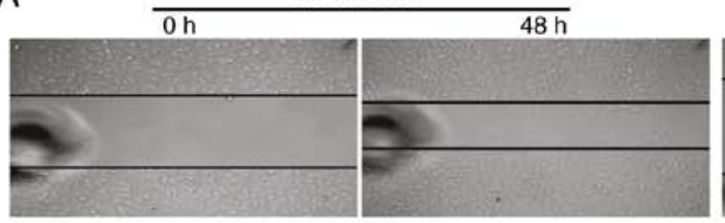

A549-SIRT7

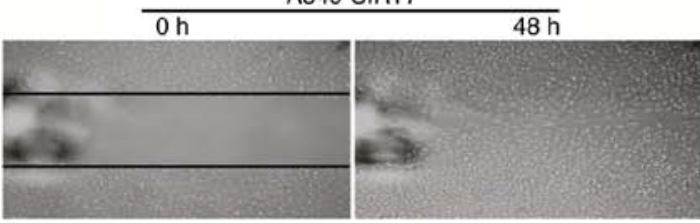

B

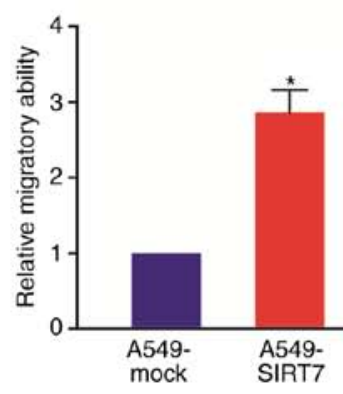

C

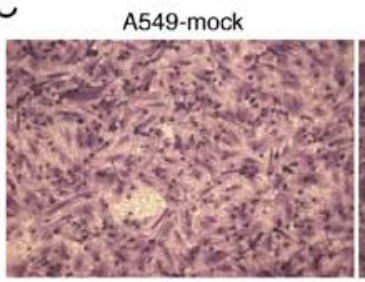

E
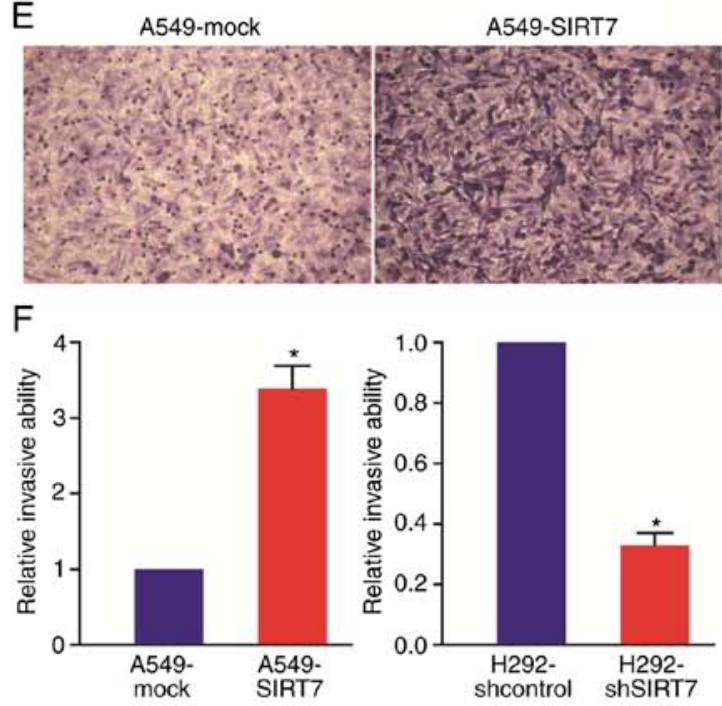

$\mathrm{G} \quad$ A549-mock

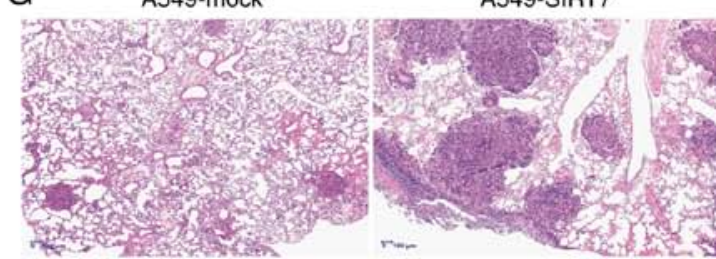

H292-shcontrol

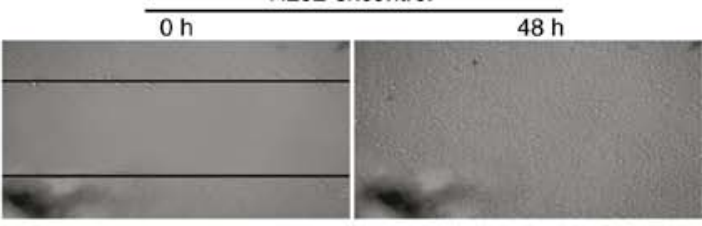

H292-shSIRT7

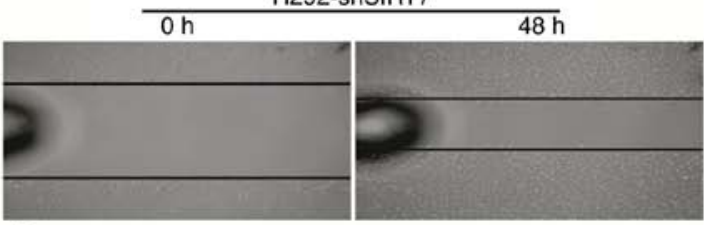

D
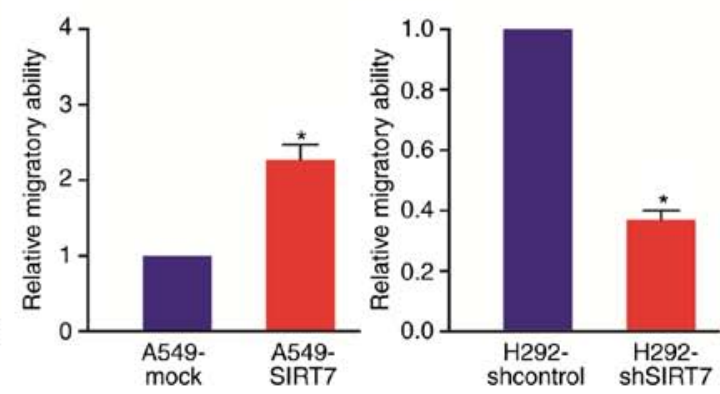

H292-shcontrol
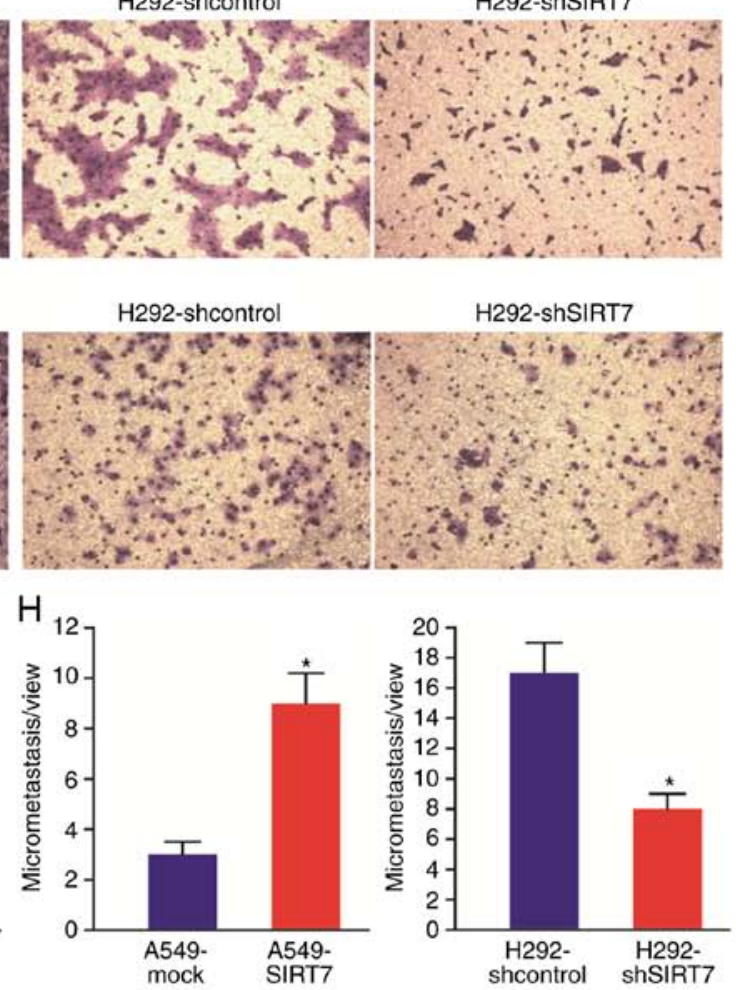

H292-shcontrol

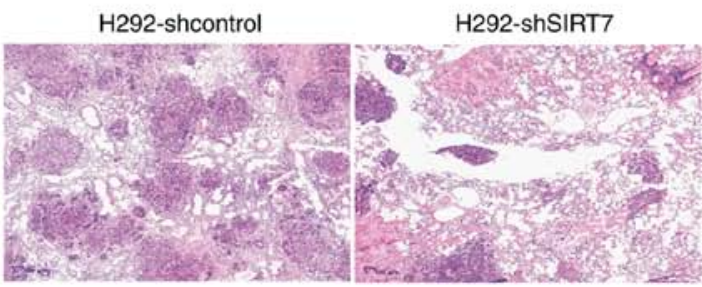

Figure 4. SIRT7 facilitates human NSCLC cell in vitro migration and invasion as well as in vivo distant lung metastasis. (A and B) Wound healing assay. (A) The representative images. (B) The relative migratory ability of A549-SIRT7 (A549-mock served as a control) and H292-shSIRT7 (H292-shcontrol served as a control) NSCLC cells. (C and D) Transwell migration assay. (C) The representative images. (D) The relative migratory ability of the aforementioned cells. (E and F) Transwell invasion assay. (E) The representative images. (F) The relative invasive ability of the aforementioned cells. (G) The representative H\&E staining images of lung tissues. (H) The number of tumor metastatic nodules in lung tissues. "P $<0.05$, Student's $t$-test, $\mathrm{n}=6$ per group. SIRT7, sirtuin 7; NSCLC, non-small cell lung cancer; H\&E, hematoxylin and eosin. 

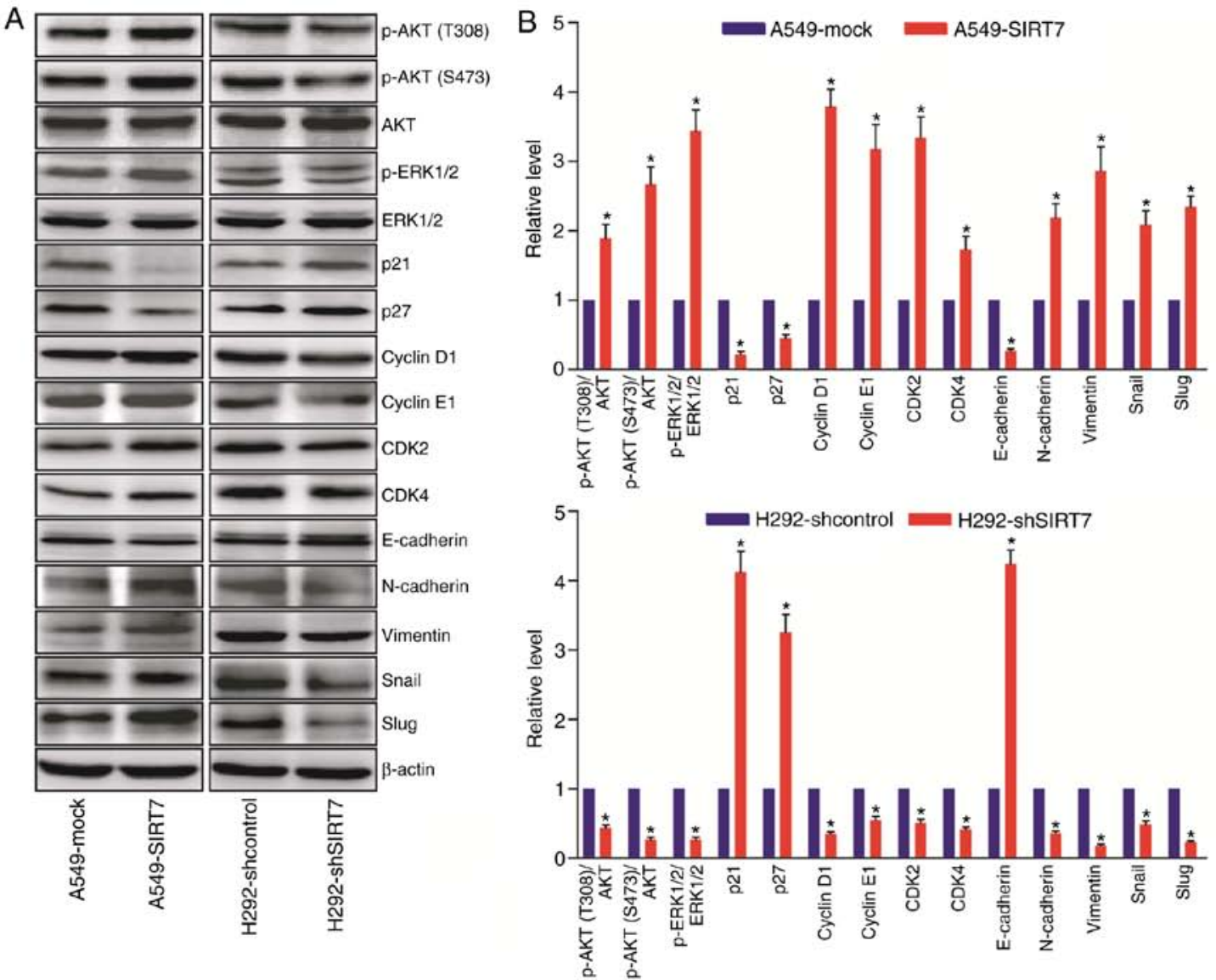

C A549-mock A549-SIRT7 A549-mock A549-SIRT7 H292-shcontrol H292-shSIRT7 H292-shcontrol H292-shSIRT7

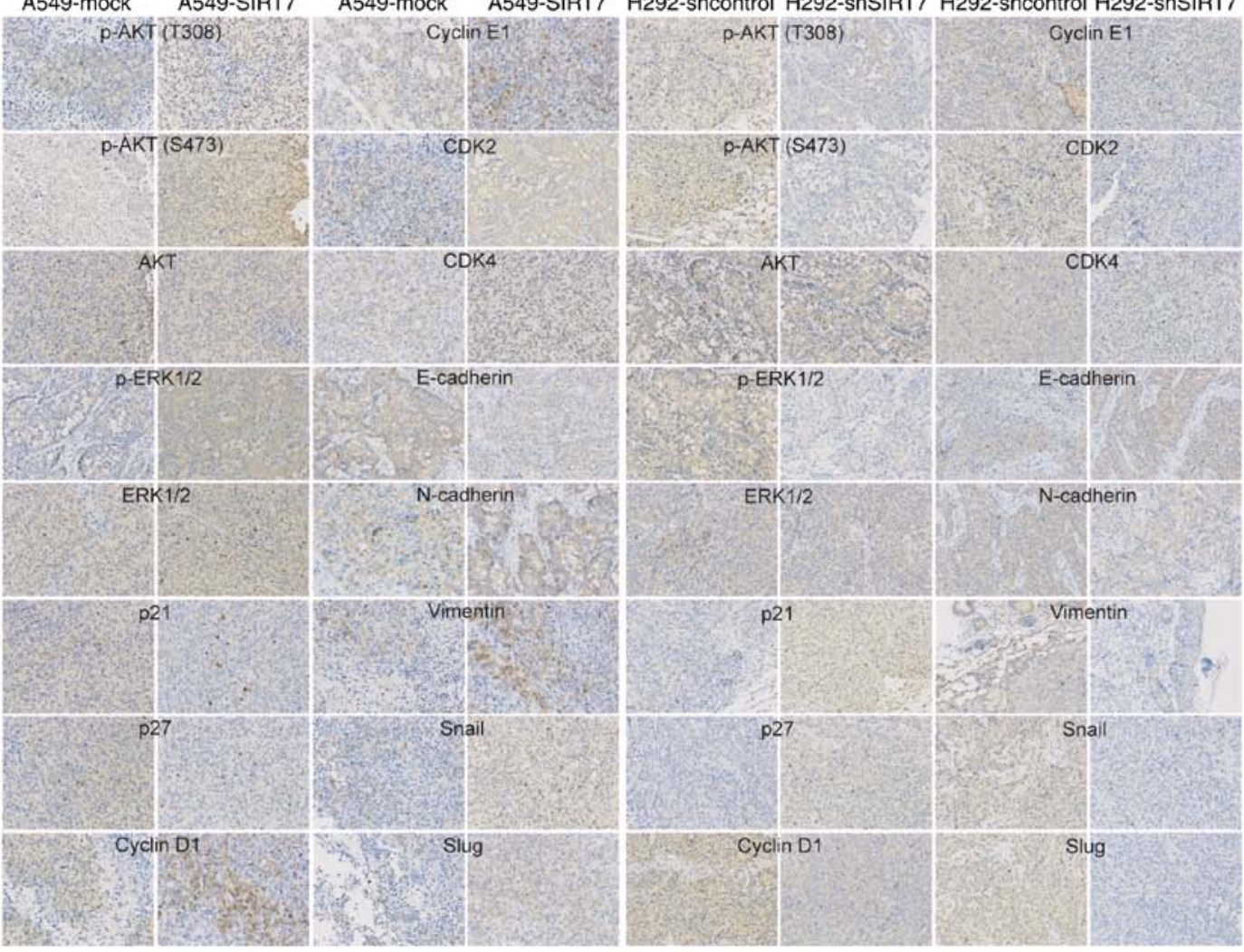

Figure 5. SIRT7 activates AKT/ERK1/2 signaling and regulates the expression of G1-phase checkpoint molecules for G1 to S transition as well as EMT molecules for EMT induction in NSCLC cells. (A and B) Western blot analysis of AKT/ERK1/2, G1-phase checkpoint and EMT molecules. (A) The representative western blot images. (B) The relative level of p-AKT (T308)/AKT, p-AKT (S473)/AKT and p-ERK1/2/ERK1/2 as well as p21, p27, cyclin D1, cyclin E1, CDK2, CDK4, E-cadherin, N-cadherin, vimentin, Snail and Slug in A549-SIRT7 (A549-mock served as a control) or H292-shSIRT7 (H292-shcontrol served as a control) NSCLC cells. "P<0.05, Student's $t$-test, $\mathrm{n}=6$ per group. (C) IHC analysis of AKT/ERK1/2, G1-phase checkpoint and EMT molecules in xenograft tumor tissues. The representative IHC images are presented. SIRT7, sirtuin 7; EMT, epithelial-mesenchymal transition; NSCLC, non-small cell lung cancer; AKT, protein kinase; ERK1/2, extracellular signal-regulated kinase 1/2; CDK, cyclin-dependent kinase; IHC, immunohistochemistry. 

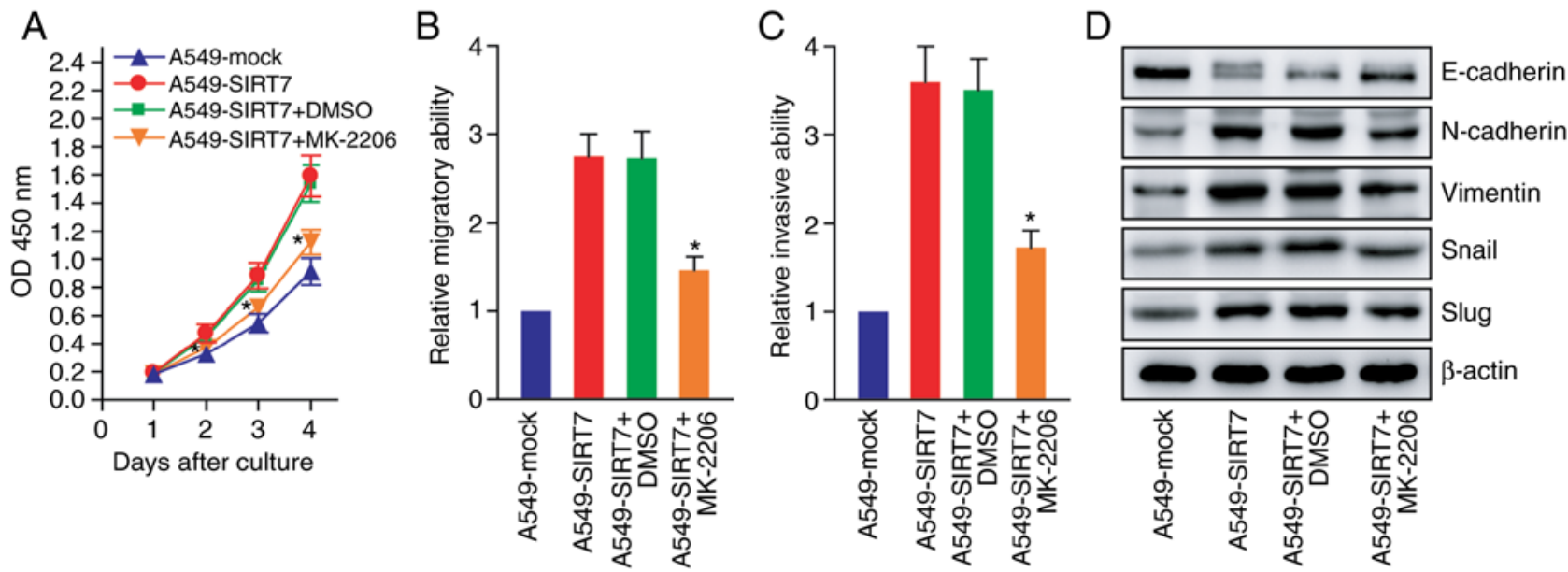

Figure 6. SIRT7 promotes NSCLC cell proliferation and EMT progression by activating AKT signaling. (A) CCK-8 assay after treatment with an AKT inhibitor (MK-2206). ${ }^{*} \mathrm{P}<0.05$ compared with A549-SIRT7 or A549-SIRT7+DMSO, two-way repeated measures ANOVA with LSD post hoc multiple comparisons, $\mathrm{n}=6$ per group. (B) Wound healing assay after treatment with the AKT inhibitor (MK-2206). * $<0.05$ compared with A549-SIRT7 or A549-SIRT7+DMSO, one-way repeated measures ANOVA with LSD post hoc multiple comparisons, $\mathrm{n}=6$ per group. (C) Transwell invasion assay after treatment with the AKT inhibitor. "P<0.05 compared with A549-SIRT7 or A549-SIRT7+DMSO, one-way repeated measures ANOVA with LSD post hoc multiple comparisons, $\mathrm{n}=6$ per group. (D) Western blot analysis of EMT markers after treatment with the AKT inhibitor. The representative western blot images are presented. SIRT7, sirtuin 7; EMT, epithelial-mesenchymal transition; NSCLC, non-small cell lung cancer; EMT, epithelial-mesenchymal transition; AKT, protein kinase B; CCK-8, Cell Counting Kit-8; DMSO, dimethylsulfoxide; ANOVA, analysis of variance; LSD, least significant difference.

favored transition of the cell cycle phase from $\mathrm{G} 1$ to $\mathrm{S}$ and accumulation of $\mathrm{S}$ phase $(\mathrm{P}<0.05)$. In contrast, in $\mathrm{H} 292$ cells, knockdown of SIRT7 induced G1-phase arrest and S-phase reduction $(\mathrm{P}<0.05)$. Therefore, the aforementioned results demonstrated the roles of SIRT7 in promoting human NSCLC cell proliferation and growth possibly by facilitating G1 to S-phase transition.

SIRT7 promotes human NSCLC cell migration and invasion in vitro as well as distant lung metastasis in vivo. To explore the roles of SIRT7 in NSCLC cell metastasis, wound healing assay and Transwell migration and invasion assays were respectively carried out to determine the migratory and invasive abilities of A549-SIRT7 vs. A549-mock and H292-shSIRT7 vs. H292-shcontrol human NSCLC cells. As revealed in Fig. 4A-D, compared with A549-mock or H292-shcontrol control cells, the migratory capability of A549-SIRT7 NSCLC cells was significantly enhanced and the migratory capability of H292-shSIRT7 NSCLC cells was weakened $(\mathrm{P}<0.05)$. Additionally, overexpression of SIRT7 in A549 NSCLC cells promoted A549 cell invasion, whereas knockdown of SIRT7 in H292 NSCLC cells impeded H292 cell invasion $(\mathrm{P}<0.05)$ (Fig. 4E and F). To further determine whether the association of SIRT7 with human NSCLC metastatic ability obtained in vitro could be replicated in vivo, the A549-SIRT7 vs. A549-mock or H292-shSIRT7 vs. H292-shcontrol cells were intravenously injected into nude mice. Six weeks later, the lung tissues of mice were harvested for H\&E analysis of lung metastatic nodules. As revealed in Fig. 4G and $\mathrm{H}$, tumor metastatic nodules in the A549-SIRT7-injected mouse-derived lungs displayed an increase compared with the A549-mock control model, whereas tumor metastatic nodules in the H292-shSIRT7-injected mouse-derived lungs exhibited a decrease compared with the H292-shcontrol control model $(\mathrm{P}<0.05)$, indicating that SIRT7 significantly strengthens the distant metastatic capacity of human NSCLC cells in vivo as well. Thus, the results demonstrated that SIRT7 can promote the metastatic potential of human NSCLC cells.

SIRT7 activates AKT and ERK1/2 signaling as well as regulates the expression of G1-phase checkpoint molecules and EMT-associated molecules in human NSCLC cells. To elucidate the molecular mechanism for SIRT7-induced promoting effects on human NSCLC cell growth and metastasis, the protein expression levels of AKT/ERK1/2 signaling molecules including p-AKT (T308/S473)/total AKT and p-ERK1/2/total ERK1/2, G1-phase checkpoint molecules including p21/27, cyclin D1/E1 and CDK2/4, and EMT-associated molecules including E/N-cadherin, vimentin, Snail and Slug in A549-SIRT7 vs. A549-mock and H292-shSIRT7 vs. H292-shcontrol human NSCLC cells were determined by western blotting. It was revealed that compared with A549-mock control cells, the expression levels of p-AKT (T308), p-AKT (S473), p-ERK1/2, cyclin D1, cyclin E1, CDK2, CDK4, N-cadherin, vimentin, Snail and Slug in A549-SIRT7 cells were significantly higher, while the expression levels of p21, p27 and E-cadherin in A549-SIRT7 cells were significantly lower $(\mathrm{P}<0.05)$ (Fig. 5A and $\mathrm{B})$. The data demonstrated that overexpression of SIRT7 activated AKT and ERK1/2 signaling, regulated the expression of G1-phase checkpoint molecules to induce G1 to S-phase transition, and promoted EMT in A549 NSCLC cells. Notably, knockdown of SIRT7 in H292 NSCLC cells exerted the opposite effects in regulating the expression of the aforementioned molecules $(\mathrm{P}<0.05)$ (Fig. 5A and B). The in vivo modulatory influence of SIRT7 on the expression of these molecules was further confirmed by IHC analysis of A549-SIRT7 vs. A549-mock and H292-shSIRT7 vs. H292-shcontrol NSCLC xenograft tumors (Fig. 5C). To further confirm the role of AKT signaling in SIRT7-mediated effects on the biological behavior of NSCLC cells and expression of EMT markers, AKT inhibition assays using MK-2206 in A549-SIRT7 human NSCLC cells was carried out. As revealed in Fig. 6A-C, inhibition of AKT signaling impaired the promoting roles of 
SIRT7 in NSCLC cell proliferation, migration and invasion $(\mathrm{P}<0.05)$. Moreover, AKT inhibition attenuated the regulating effects of SIRT7 on the expression of EMT markers such as E-cadherin, N-cadherin, vimentin, Snail and Slug in NSCLC cells (Fig. 6D). The findings indicated that SIRT7 can promote human NSCLC cell growth and metastasis probably by activating $\mathrm{AKT}$ and ERK1/2 signaling as well as regulating the expression of G1-phase checkpoint molecules to promote G1 to S-phase transition and EMT-associated molecules to facilitate EMT.

\section{Discussion}

As the newest member of sirtuin family, SIRT7 plays essential roles and exhibits dual functions in tumorigenesis and progression $(13,33)$. The present study demonstrated that SIRT7 was increased in human lung cancer tissues and NSCLC cell lines, and there was a positive correlation between SIRT7 expression level in tumor tissues and malignant clinicopathological characteristics (large tumor size, high pathologic stage and positive lymph node metastasis) of lung cancer patients. Using human NSCLC cell and xenograft mouse models, it was revealed that SIRT7 overexpression promoted NSCLC cell in vitro and in vivo proliferation/growth, G1 to S-phase transition, migration and invasion as well as in vivo distant lung metastasis, whereas SIRT7 knockdown suppressed these processes. Furthermore, SIRT7 overexpression strengthened activation of AKT/ERK1/2 signaling as well as regulated the expression of G1-phase checkpoint molecules to promote G1 to S-phase transition and EMT-associated molecules to facilitate EMT in NSCLC cells.

The abnormal activation of the phosphatidylinositol 3-kinase (PI3K)-AKT and mitogen-activated protein kinase (MAPK)-ERK1/2 pathway is considered to contribute to carcinogenesis and cancer progression (34-36). A high level of phosphorylated AKT and ERK1/2 has also been revealed to frequently occur in human NSCLC, affirming them as potential therapeutic targets for NSCLC (37). Previous studies have revealed the role of SIRT7 in modulating AKT and ERK1/2 signaling. Vakhrusheva et al (38) revealed the increase of phosphorylated AKT in the heart of SIRT7 ${ }^{-/-}$mice. Under energy stress conditions, SIRT7 can suppress AKT activation via deacetylation of FKBP51 and subsequent enhanced interaction between PHLPP and AKT, thereby sensitizing breast cancer cells to cytotoxic agents (39). Controversially, SIRT7 has been revealed to promote activation of AKT and p70S6K1 in thyroid cancer via the DBC1/SIRT1 axis (40). In addition, SIRT7 was able to activate the MAPK-ERK1/2 pathway as well as increase ERK1/2 phosphorylation in colorectal cancer (16) and hepatocellular carcinoma (41). In accordance with these findings $(16,40,41)$, the present study revealed that AKT and ERK1/2 phosphorylation were increased in SIRT7-overexpressed NSCLC cells, whereas their levels were reduced in SIRT7-knocked down NSCLC cells. The data indicated that SIRT7 was capable of activating AKT/ERK1/2 signaling in NSCLC cells, which may contribute to SIRT7-induced promoting effects on NSCLC cell growth and metastasis.

Cell cycle dysregulation control may result in tumorigenesis and cancer development (42). Proteins involved in cell cycle regulation include cyclins, cyclin-dependent kinases (CDKs) and CDK inhibitors (42). The main G1-phase checkpoint molecules include cyclin A, cyclin D and cyclin E cyclins, CDK2, CDK4 and CDK6 CDKs, and p21 and p27 CDK inhibitors (42). Among these elements, p21 can suppress the activity of the cyclin E/A-CDK2 complex, whereas p27 can suppress the activity of the cyclin D-CDK4/6 complex, leading to cell cycle arrest in the G1 phase (42). In order to explore the cellular mechanism for SIRT7-induced NSCLC cell growth promotion, cell cycle analysis in SIRT7-overexpressed and SIRT7-knocked down NSCLC cells was performed by flow cytometry. It was demonstrated that SIRT7 overexpression accelerated cell cycle progression by favoring cell cycle phase transition from G1 to S in NSCLC cells, while SIRT7 knockdown induced G1-phase arrest. The effects of SIRT7 on the expression of G1-phase checkpoint molecules including p21, p27, cyclin D1, cyclin E1, CDK2 and CDK4 were then detected, demonstrating that overexpression of SIRT7 upregulated cyclin D1, cyclin E1, CDK2 and CDK4, and downregulated p21 and p27, whereas knockdown of SIRT7 exhibited the opposite regulatory effects. Previous studies $(18,19)$ have revealed that SIRT7 can promote tumor cell cycle progression via downregulation of p21 and upregulation of cyclin D1 and CDK2, which are consistent with the present results. Thus, it is concluded that SIRT7 promotes human NSCLC proliferation and growth to a great extent via accelerating G1 to S-phase transition by regulating the expression of G1-phase checkpoint molecules.

EMT endows epithelial-derived tumor cells with enhanced migratory, invasive and metastatic ability and is involved in cancer metastasis $(43,44)$. Main steps can be observed in EMT progression. For example, the epithelial cell-cell junctions are disintegrated, the apical-basal polarity is lost, the front-rear polarity is acquired, the cytoskeletal architecture is reorganized and the cell shape is altered. It can also be detected that the expression of E-cadherin epithelial marker is downregulated and the expression of $\mathrm{N}$-cadherin and vimentin mesenchymal markers is upregulated $(43,44)$. EMT can be driven by Snail/Slug, Twist (Twist1)/Twist 2 and Zeb1/Zeb2 transcription factors with the ability to suppress E-cadherin and activate $\mathrm{N}$-cadherin and vimentin directly or indirectly $(43,44)$. Previous studies have revealed the role of SIRT7 in activation of EMT in several cancers $(16,20,45,46)$. To further clarify the association between SIRT7 and EMT in NSCLC cells, the present study determined the effects of SIRT7 overexpression or knockdown on the expression of EMT-related molecules in human NSCLC cells. As anticipated, overexpression of SIRT7 downregulated E-cadherin as well as upregulated Snail, Slug, N-cadherin and vimentin. However, knockdown of SIRT7 exhibited the opposite effects. The present findings indicated that SIRT7 promoted EMT of NSCLC cells, which may be involved in SIRT7-stimulated NSCLC metastasis. Although the exact mechanism by which SIRT7 regulates EMT-associated molecules in NSCLC cells remains elusive, the SIRT7-induced upregulation of Snail and Slug may be responsible for SIRT7-mediated E-cadherin reduction or $\mathrm{N}$-cadherin and vimentin increase. Active glycogen synthase kinase $3 \beta$ (GSK3 $\beta$ ) has been revealed to be capable of phosphorylating Snail/Slug and increasing $\beta$-transducin repeat-containing protein $(\beta \mathrm{TrCP})$ - or carboxyl terminus of Hsc70-interacting protein (CHIP)-induced Snail 
or Slug ubiquitylation, leading to proteasomal degradation of Snail and Slug (44,47-49). It has also been demonstrated that active AKT and ERK1/2 can phosphorylate GSK3 $\beta$ at Ser9 residue and inactivate activity of GSK3 $\beta$ (49,50). Therefore, SIRT7-induced activation of AKT/ERK1/2 signaling may participate in SIRT7-induced upregulation of Snail and Slug in NSCLC cells by positively modulating their stability through the AKT/ERK1/2-GSK3 $\beta$-Snail/Slug axis. Notably, emerging evidence has revealed that SIRT7 can directly bind to the promoter of E-cadherin to promote H3K18Ac deacetylation, resulting in inhibition of E-cadherin transcription $(45,46)$, which prompted us to infer that SIRT7 may also directly suppress E-cadherin expression of NSCLC cells in a chromatin-remodeling manner.

Collectively, the present study demonstrated that SIRT7 was capable of promoting human NSCLC cell growth and metastasis possibly by activating AKT/ERK1/2 signaling as well as favoring cell cycle phase transition from $\mathrm{G} 1$ to $\mathrm{S}$ via regulation of G1-phase checkpoint molecules and inducing EMT. SIRT7 functions as an oncogene and could be a novel therapeutic target in human NSCLC.

\section{Acknowledgements}

Not applicable.

\section{Funding}

The present study was supported by grants from the National Natural Science Foundation of China (NNSFC) (nos. 81772645, 81572992, 81602704 and 81372443), the Science and Technology Department of Jiangsu Province (nos. BY2015039-03 and BL2014039), the Health and Family Planning Commission of Jiangsu Province (no. LGY2016030), the Suzhou Institute of Biomedical Engineering and Technology of the Chinese Academy of Sciences (no. Y851411105), the Natural Science Foundation of the Jiangsu Higher Education Institutions of China (no. 16KJB320012), the Beijing Xisike Clinical Oncology Research Foundation (no. Y-MX2016-017), the Pushin HK Jiangsu Medical Technology Ltd., Inc. (no. P112200315) and the Wu Jieping Medical Foundation (no. P112200914).

\section{Availability of data and materials}

The datasets used during the present study are available from the corresponding author upon reasonable request.

\section{Authors' contributions}

This study was conceived and designed by YX, MT, CX and YZ. The clinical tissue specimens were collected by CX, DZ and CL. The experiments and the data analyses were completed by YZ and XY. Other authors including RC, QG, DZ and YQ also participated in the data analyses of this study. The manuscript was written by $\mathrm{YZ}$ and $\mathrm{XY}$, and supervised by YX and MT. All authors read and approved the manuscript and agree to be accountable for all aspects of the research in ensuring that the accuracy or integrity of any part of the work are appropriately investigated and resolved.

\section{Ethics approval and consent to participate}

The present study was approved by the Ethics Committee of The First Affiliated Hospital of Soochow University (IRB no. 2016128). All patients had provided written informed consent prior to obtaining the samples. All animal experiments were approved by the Animal Research Ethics Committee of Soochow University (IRB no. A201809059).

\section{Patient consent for publication}

Not applicable.

\section{Competing interests}

The authors declare that there are no competing interests.

\section{References}

1. Torre LA, Bray F, Siegel RL, Ferlay J, Lortet-Tieulent J and Jemal A: Global cancer statistics, 2012. CA Cancer J Clin 65: 87-108, 2015.

2. Zappa C and Mousa SA: Non-small cell lung cancer: Current treatment and future advances. Transl Lung Cancer Res 5: 288-300, 2016.

3. Allemani C, Matsuda T, Di Carlo V, Harewood R, Matz M, Nikšić M, Bonaventure A, Valkov M, Johnson CJ, Estève J, et al: Global surveillance of trends in cancer survival 2000-14 (CONCORD-3): Analysis of individual records for 37513 025 patients diagnosed with one of 18 cancers from 322 population-based registries in 71 countries. Lancet 391: 1023-1075, 2018.

4. Chen W, Zheng R, Baade PD, Zhang S, Zeng H, Bray F, Jemal A, $\mathrm{Yu}$ XQ and He J: Cancer statistics in China, 2015. CA Cancer J Clin 66: 115-132, 2016.

5. Finkel T, Deng CX and Mostoslavsky R: Recent progress in the biology and physiology of sirtuins. Nature 460: 587-591, 2009.

6. Chalkiadaki A and Guarente L: The multifaceted functions of sirtuins in cancer. Nat Rev Cancer 15: 608-624, 2015.

7. Michishita E, Park JY, Burneskis JM, Barrett JC and Horikawa I: Evolutionarily conserved and nonconserved cellular localizations and functions of human SIRT proteins. Mol Biol Cell 16: 4623-4635, 2005.

8. Houtkooper RH, Pirinen E and Auwerx J: Sirtuins as regulators of metabolism and healthspan. Nat Rev Mol Cell Biol 13: 225-238, 2012.

9. Ford E, Voit R, Liszt G, Magin C, Grummt I and Guarente L: Mammalian Sir2 homolog SIRT7 is an activator of RNA polymerase I transcription. Genes Dev 20: 1075-1080, 2006.

10. Chen S, Blank MF, Iyer A, Huang B, Wang L, Grummt I and Voit R: SIRT7-dependent deacetylation of the U3-55k protein controls pre-rRNA processing. Nat Commun 7: 10734, 2016.

11. Tsai YC, Greco TM and Cristea IM: Sirtuin 7 plays a role in ribosome biogenesis and protein synthesis. Mol Cell Proteomics 13: 73-83, 2014.

12. Barber MF, Michishita-Kioi E, Xi Y, Tasselli L, Kioi M, Moqtaderi Z, Tennen RI, Paredes S, Young NL, Chen K, et al: SIRT7 links H3K18 deacetylation to maintenance of oncogenic transformation. Nature 487: 114-118, 2012.

13. Paredes S, Villanova L and Chua KF: Molecular pathways: Emerging roles of mammalian Sirtuin SIRT7 in cancer. Clin Cancer Res 20: 1741-1746, 2014.

14. Geng Q, Peng H, Chen F, Luo R and Li R: High expression of Sirt7 served as a predictor of adverse outcome in breast cancer. Int J Clin Exp Pathol 8: 1938-1945, 2015.

15. de Nigris F, Cerutti J, Morelli C, Califano D, Chiariotti L, Viglietto G, Santelli G and Fusco A: Isolation of a SIR-like gene, SIR-T8, that is overexpressed in thyroid carcinoma cell lines and tissues. Br J Cancer 86: 917-923, 2002.

16. Yu H, Ye W, Wu J, Meng X, Liu RY, Ying X, Zhou Y, Wang H, Pan C and Huang W: Overexpression of sirt7 exhibits oncogenic property and serves as a prognostic factor in colorectal cancer. Clin Cancer Res 20: 3434-3445, 2014. 
17. Shen X, Li P, Xu Y, Chen X, Sun H, Zhao Y, Liu M and Zhang W: Association of sirtuins with clinicopathological parameters and overall survival in gastric cancer. Oncotarget 8: 74359-74370, 2017.

18. Kim JK, Noh JH, Jung KH, Eun JW, Bae HJ, Kim MG, Chang YG, Shen Q, Park WS, Lee JY, et al: Sirtuin7 oncogenic potential in human hepatocellular carcinoma and its regulation by the tumor suppressors MiR-125a-5p and MiR-125b. Hepatology 57: $1055-1067,2013$

19. Li W, Sun Z, Chen C, Wang L, Geng Z and Tao J: Sirtuin7 has an oncogenic potential via promoting the growth of cholangiocarcinoma cells. Biomed Pharmacother 100: 257-266, 2018.

20. Haider R, Massa F, Kaminski L, Clavel S, Djabari Z, Robert G, Laurent K, Michiels JF, Durand M, Ricci JE, et al: Sirtuin 7: A new marker of aggressiveness in prostate cancer. Oncotarget 8: 77309-77316, 2017.

21. Han Y, Liu Y, Zhang H, Wang T, Diao R, Jiang Z, Gui Y and Cai Z: Hsa-miR-125b suppresses bladder cancer development by down-regulating oncogene SIRT7 and oncogenic long non-coding RNA MALAT1. FEBS Lett 587: 3875-3882, 2013.

22. Wang $X$ and Song Y: MicroRNA-340 inhibits the growth and invasion of angiosarcoma cells by targeting SIRT7. Biomed Pharmacother 103: 1061-1068, 2018.

23. Kiran S, Oddi V and Ramakrishna G: Sirtuin 7 promotes cellular survival following genomic stress by attenuation of DNA damage, SAPK activation and p53 response. Exp Cell Res 331: 123-141, 2015.

24. Tang M, Lu X, Zhang C, Du C, Cao L, Hou T, Li Z, Tu B, Cao Z, Li Y, et al: Downregulation of SIRT7 by 5-fluorouracil induces radiosensitivity in human colorectal cancer. Theranostics 7: 1346-1359, 2017.

25. Lai CC, Lin PM, Lin SF, Hsu CH, Lin HC, Hu ML, Hsu CM and Yang MY: Altered expression of SIRT gene family in head and neck squamous cell carcinoma. Tumour Biol 34: 1847-1854, 2013.

26. McGlynn LM, McCluney S, Jamieson NB, Thomson J, MacDonald AI, Oien K, Dickson EJ, Carter CR, McKay CJ and Shiels PG: SIRT3 \& SIRT7: Potential novel biomarkers for determining outcome in pancreatic cancer patients. PLoS One 10 e0131344, 2015.

27. Li W,Zhu D and Qin S: SIRT7 suppresses the epithelial-to-mesenchymal transition in oral squamous cell carcinoma metastasis by promoting SMAD4 deacetylation. J Exp Clin Cancer Res 37: $148,2018$.

28. Tang X, Shi L, Xie N, Liu Z, Qian M, Meng F, Xu Q, Zhou M, Cao X, Zhu WG and Liu B: SIRT7 antagonizes TGF- $\beta$ signaling and inhibits breast cancer metastasis. Nat Commun 8: 318, 2017.

29. Shi H, Ji Y,Zhang D, Liu Y and Fang P: MicroRNA-3666-induced suppression of SIRT7 inhibits the growth of non-small cell lung cancer cells. Oncol Rep 36: 3051-3057, 2016.

30. Jiang Y, Han Z, Wang Y and Hao W: Depletion of SIRT7 sensitizes human non-small cell lung cancer cells to gemcitabine therapy by inhibiting autophagy. Biochem Biophys Res Commun 506: 266-271, 2018

31. Qian F, Hu Q, Tian Y, Wu J, Li D, Tao M, Qin L, Shen B and Xie Y: ING4 suppresses hepatocellular carcinoma via a $\mathrm{NF}-\kappa \mathrm{B} / \mathrm{miR}-155 / \mathrm{FOXO}$ a signaling axis. Int J Biol Sci 15: 369-385, 2019.

32. Schmittgen TD and Livak KJ: Analyzing real-time PCR data by the comparative C(T) method. Nat Protoc 3: 1101-1108, 2008.

33. Li L and Bhatia R: The controversial role of Sirtuins in tumorigenesis-SIRT7 joins the debate. Cell Res 23: 10-12, 2013.

34. Fruman DA, Chiu H, Hopkins BD, Bagrodia S, Cantley LC and Abraham RT: The PI3K pathway in human disease. Cell 170 605-635, 2017
35. Thorpe LM, Yuzugullu H and Zhao JJ: PI3K in cancer: Divergent roles of isoforms, modes of activation and therapeutic targeting. Nat Rev Cancer 15: 7-24, 2015.

36. Dhillon AS, Hagan S, Rath O and Kolch W: MAP kinase signalling pathways in cancer. Oncogene 26: 3279-3290, 2007.

37. Ciuffreda L, Incani UC, Steelman LS, Abrams SL, Falcone I, Curatolo AD, Chappell WH, Franklin RA, Vari S, Cognetti F, et al: Signaling intermediates (MAPK and PI3K) as therapeutic targets in NSCLC. Curr Pharm Des 20: 3944-3957, 2014.

38. Vakhrusheva O, Smolka C, Gajawada P, Kostin S, Boettger T, Kubin T, Braun T and Bober E: Sirt7 increases stress resistance of cardiomyocytes and prevents apoptosis and inflammatory cardiomyopathy in mice. Circ Res 102: 703-710, 2008.

39. Yu J, Qin B, Wu F, Qin S, Nowsheen S, Shan S, Zayas J, Pei H, Lou $\mathrm{Z}$ and Wang L: Regulation of serine-threonine kinase Akt activation by NAD+-dependent deacetylase SIRT7. Cell Rep 18: 1229-1240, 2017.

40. Li H, Tian Z, Qu Y, Yang Q, Guan H, Shi B, Ji M and Hou P: SIRT7 promotes thyroid tumorigenesis through phosphorylation and activation of Akt and p70S6K1 via DBC1/SIRT1 axis. Oncogene 38: 345-359, 2019.

41. Liu X, Yang L, Tu J, Cai W, Zhang M, Shou Z, Yao Y and Xu Q: microRNA-526b servers as a prognostic factor and exhibits tumor suppressive property by targeting Sirtuin 7 in hepatocellular carcinoma. Oncotarget 8: 87737-87749, 2017.

42. Massagué J: G1 cell-cycle control and cancer. Nature 432: 298-306, 2004.

43. Brabletz T, Kalluri R, Nieto MA and Weinberg RA: EMT in cancer. Nat Rev Cancer 18: 128-134, 2018.

44. Lamouille S, Xu J and Derynck R: Molecular mechanisms of epithelial-mesenchymal transition. Nat Rev Mol Cell Biol 15: 178-196, 2014.

45. Deng Z, Wang X, Long X, Liu W, Xiang C, Bao F and Wang D: Sirtuin 7 promotes colorectal carcinoma proliferation and invasion through the inhibition of E-cadherin. Exp Ther Med 15: 2333-2342, 2018.

46. Malik S, Villanova L, Tanaka S, Aonuma M, Roy N, Berber E, Pollack JR, Michishita-Kioi E and Chua KF: SIRT7 inactivation reverses metastatic phenotypes in epithelial and mesenchymal tumors. Sci Rep 5: 9841, 2015.

47. Zhou BP, Deng J, Xia W, Xu J, Li YM, Gunduz M and Hung MC: Dual regulation of Snail by GSK-3beta-mediated phosphorylation in control of epithelial-mesenchymal transition. Nat Cell Biol 6: 931-940, 2004.

48. Schlessinger K and Hall A: GSK-3beta sets Snail's pace. Nat Cell Biol 6: 913-915, 2004

49. Kao SH, Wang WL, Chen CY, Chang YL, Wu YY, Wang YT, Wang SP, Nesvizhskii AI, Chen YJ, Hong TM and Yang PC: GSK $3 \beta$ controls epithelial-mesenchymal transition and tumor metastasis by CHIP-mediated degradation of Slug. Oncogene 33: 3172-3182, 2014

50. Zhu P, Lv J, Yang Z, Guo L, Zhang L, Li M, Han W, Chen X, Zhuang $\mathrm{H}$ and Lu F: Protocadherin 9 inhibits epithelial-mesenchymal transition and cell migration through activating GSK-3 $\beta$ in hepatocellular carcinoma. Biochem Biophys Res Commun 452: 567-574, 2014.

This work is licensed under a Creative Commons Attribution-NonCommercial-NoDerivatives 4.0 International (CC BY-NC-ND 4.0) License. 